\title{
Investigation of Passive Oscillations of Flexible Splitter Plates Attached to a Circular Cylinder
}

\author{
Rezvan Abdi ${ }^{*}$, Niki Rezazadeh ${ }^{1}$, Meisam Abdi $^{2}$ \\ *Rezvan_Abdi@yahoo.com \\ ${ }^{1}$ Faculty of Engineering, Hakim Sabzevari University, Sabzevar, Iran \\ ${ }^{2}$ Faculty of Technology, De Montfort University, Leicester, UK
}

\begin{abstract}
This paper presents a numerical study to address wake control of a circular cylinder subjected to two-dimensional laminar flow regime using single and multiple flexible splitter plates attached to the cylinder. Three different cases are presented in the study, covering cylinders with one, two and three horizontally attached splitter plates while the locations of the plates around the cylinders are varied. The length of the splitter plates was equal to the cylinder diameter and Reynolds number was 100. Due to the flexibility of the plates, the problem was modeled as a Fluid-Structure Interaction (FSI) problem and the commercial finite element software, Comsol Multiphysics, was utilized to solve this problem using Arbitrary Lagrangian-Eulerian (ALE) method. Vortex shedding frequency and fluid forces acting on the cylinder are investigated, along with a comprehensive parametric study to identify the optimum arrangement of the plates for maximum drag reduction and maximum vortex shedding frequency reduction. The numerical results associated to the flexible splitter plates are also compared with the corresponding rigid splitter plate cases investigated in a previous study. Moreover, the tip amplitude of the plates and the maximum strains were measured in order to find an optimum position for placing a piezoelectric polymer to harvest energy from the flow.
\end{abstract}

Keywords: fluid-structure interaction, Arbitrary Lagrangian-Eulerian, flexible splitter plate, vortex shedding, drag, Strouhal number.

\section{Introduction}

Many engineering applications require control of fluid forces for certain needs such as vibration suppression, drag reduction and lift enhancement (Wu et al, 2014). Examples include heat exchangers, marine structures, bridges and power transmission lines (Sudhakar and Vengadesan, 2012). Flow past a circular cylinder has been extensively studied as a representative example of flow over a bluff body (Wu et al, 2014).

Previous works have shown that the formation of vortex shedding behind a cylinder can be effectively reduced and suppressed by the use of splitter plates (Kwon and Choi, 1996). Roshko (1954) initiated the research on the application of splitter plates in wake control by investigating the effect of the length of a rigid splitter plate $(L)$ on cylinder wake. In another study, a detached rigid splitter plate of length $L=D$ (where $D$ is the cylinder diameter) was used to control the cylinder wake (Roshko, 1955). Gerrard (1966) studied the effect of the length of a rigid splitter plate on cylinder wake at $R e=2 \times 10^{4}$. It was observed that by increasing the length of the splitter plate within $0 \leq L / D \leq 1$, the Strouhal number (St) (a dimensionless number describing oscillating flow mechanisms) decreased. However, further 
increase in the plate length $(L / D>1)$ resulted in a gradual increase in St, suggesting that the minimum St was achievable when $L / D=1$. Moreover, the frequency of vortex shedding was inversely proportional to the vortex formation length immediately downstream of the bluff body.

Other studies investigated the effect of Reynolds number, length of the rigid plates, shape of the bluff body and distance between plate and bluff body on flow quantities of the cylinder (Bearman, 1965; Apelt et al., 1973; Apelt and West, 1975; Unal and Rockwell (1988); Nakamura, 1996; Ozono, 2003; Akilli et al, 2005). Abdi et al (2017) investigated the wake control of a circular cylinder using multiple rigid splitter plates at $\mathrm{Re}=100$. It was observed that the use of dual-splitters and tri-splitters led to further decrease in drag and vortex shedding frequency compared to single rigid plate case. Moreover, the attachment angle had a crucial effect on the control efficiency.

The utilization of a rigid splitter plate behind a cylinder prevents any communication between the two shear layers downstream of the cylinder (Shukla, 2009). Recent studies investigated alternative cases where the communication between the two shear layers is not totally inhibited. Cardell (1993) studied the effect of permeability of the control plate on flow quantities of a cylinder. The same base pressure as the cylinder with an attached rigid splitter plate was observed when a rigid control plate with low permeability was utilized whereas by increasing the permeability, the base pressure smoothly reached that of the plain cylinder. More recently, Shukla et al. (2009) investigated the influence of attaching hinged-rigid splitter plate to a cylinder on the cylinder wake. It was observed that the splitter plate oscillated due to the pressure difference across the plate and the amplitude of oscillations reached a maximum value of $0.45 \mathrm{D}$ at the free end. Hinged splitter plates are also studied by Assi et al. (2009) and Gu et al. (2012).

Unlike the rigid splitter plates which have been extensively studied, there are only a few studies applying flexible splitter plates for wake control. In the case of flexible splitter plates, the local difference in pressure and viscous shear stresses on the plates' surfaces can cause periodic deformation of the plates along their length. The flow field will also be affected by the plate displacements, resulting in a coupling process between the fluid and the structure which is known as fluid-structure interaction (FSI). Consideration of elastic deformation of the splitter plates can provide great practical importance. For instance, this system can be used to harvest energy from the flow by means of piezoelectric membranes. Extensive studies have been done in this area, e.g. Allen and Smits (2001), Taylor et al (2001) and Akaydin et al (2010). Moreover, some studies investigated the effect of flexibility on propulsive velocity. Lee and Lee (2013) studied a flapping flexible plate (without the cylinder) moved with propulsive velocity in quiescent fluid and found that the use of a flexible plate improves the propulsive velocity (when compared with rigid plate) to create optimal propulsion.

With the development of computational power in recent decades, numerical simulation techniques have become a powerful tool for solving the FSI problems. FSI problems are mathematically represented by coupling fluid dynamics and structural mechanics equations to get solved simultaneously. A number of different approaches can be used for solving FSI problems, such as Immersed Boundary Method (IBM), Lattice Boltzmann Methods (LBM) and Arbitrary Lagrangian-Eulerian (ALE) method. Sudhakar and Vengadesan (2012) studied the vortex shedding characteristics and the drag forces acting on a circular cylinder attached to an oscillating rigid splitter plate at $R e=100$ using the concept of Immersed Boundary Method (IBM). The rigid plate was forced to oscillate by varying the frequency and amplitude of plate oscillations. Three different patterns of vortex shedding were observed in the wake of the circular cylinder: normal shedding, chain of vortices and shedding from 
splitter plate. In a similar study, Wu et al (2014) investigated effects of detached flexible splitter plate located on both upstream and downstream of the cylinder using a coupled Immersed Boundary-Lattice Boltzmann Method (IB-LBM). A simulation of the interaction between multiple elastic structures and a viscous incompressible fluid was performed by Tian et al (2011). They introduced a modified penalty approach into the IB-LBM to simulate the moving boundaries. However, this approach introduced artificial spring parameters to calculate the distributed force and caused many uncertainties in the results (Yuan et al, 2014).

Among various numerical methods to solve the Navier-Stokes equation in a fluid domain with moving boundaries, ALE method has been found as a successful approach (Souli et al, 2000). ALE method employs a moving mesh strategy which allows adaptive mesh refinement with the movement of a structure. This method is found to benefit from high accuracy due to its boundary adaptability (Lee and Lee, 2013). The ALE method was used by Sawada and Hisada (2007) to study the two-dimensional flag-in-wind problem. They investigated the effects of a number of representative parameters on the amplitude and frequency of oscillations to reveal the underlying mechanism of flag flapping.

As discussed above, the majority of previous works related to control and stabilization of wake flow have focused on the use of rigid splitter plates mainly in a fixed angular position for wake control. The aim of this paper is to investigate the effect of flexible splitter plates on wake control of a circular cylinder by performing a two-dimensional (2D) numerical study. The plates exhibit periodic deformation which results in a complicated FSI problem. The novelty of the study is that it investigates the effect of multiple flexible plates attached to a cylinder on the cylinder wake, by comparing three different cases: single-splitter, dualsplitters, and tri-splitters. Moreover, the effect of the location of horizontal plates (attachment angle) on the cylinder wake is studied in the paper. The variations of drag coefficient, St, root-mean-square (r.m.s) value of the fluctuating lift coefficient, tip displacement and strain along the plates' length are also investigated. In all cases, it is considered that $L / D=1$, since the St reaches its minimum value at the splitter plate ratio of one (Gerrard, 1966; Apelt et al, 1973). Based on the cylinder diameter, the Reynolds number was fixed at 100 . Since the three-dimensionality of the flow evolves at $R e>160$ for a flow over a circular cylinder (Mansy et al 1994), the utilization of two-dimensional Navier-Stokes equations can provide a physically appropriate solution for the presented problem (Sudhakar and Vengadesan, 2012). The FSI module provided by the commercial finite element software, Comsol Multiphysics (COMSOL Inc., Stockholm, Sweden) is utilized to solve the problem using the ALE method. The next sections of the paper present the problem statement followed by the numerical methodology used to solve the governing equations, detailed results and discussion.

\section{Problem statement}

Free oscillations of flexible splitter plates attached to the rear surface of a circular cylinder is considered. Three different cases are presented, including the cylinder with one flexible splitter plate (single splitter), two flexible splitter plates (dual-splitters) and three flexible splitter plates (tri-splitters) as illustrated in figure 1 . The thickness $(h)$ of all plates was equal to $0.03 \mathrm{D}$ in all cases and the location of the horizontal plates behind the cylinder was defined by attachment angle $\left(\theta_{p}\right)$. This is the angle between the wake centerline and a straight line crossing the centre of the cylinder and the attachment point. $\theta_{p}$ was varied between $\theta_{p}=0^{\circ}$ and $\theta_{p}=90^{\circ}$. It was assumed that the attachment points of dual-splitters and tri-splitters are symmetric with respect to $x$ axis. 


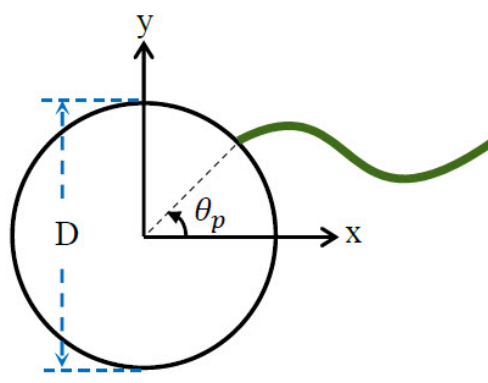

(a)

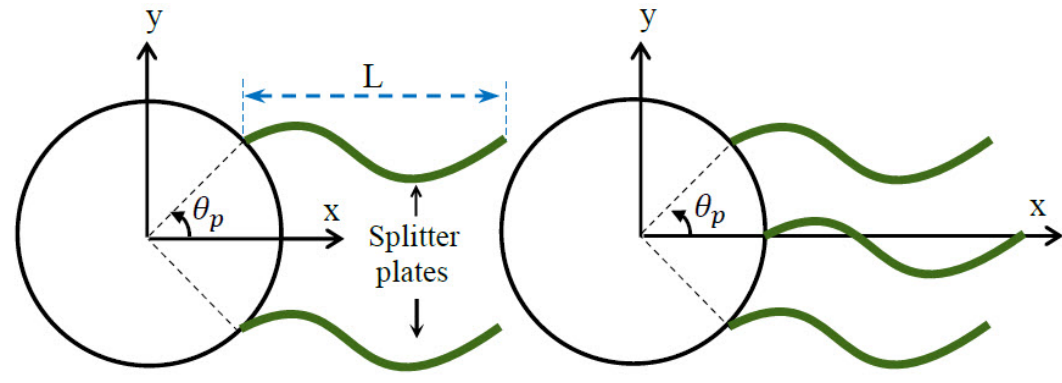

(b) (c)

Figure 1: Schematic diagram of a cylinder with attached flexible splitter plates. (a) single splitter (b) dual-splitters (c) tri-splitters.

A 2D rectangular computational domain defined by $-30 D \leq x \leq 50 D$ and $-30 D \leq y \leq$ $30 D$ was used (figure 2) and the centre of the cylinder was placed at the origin $(x=0, y=$ 0 ). The effect of the outlet boundary and the blockage effect were negligible in this problem as the size of the domain was large enough in both flow and cross-flow directions. Figure 2 demonstrates the time dependent boundary conditions which are given as:

1) The cylinder surface and the plates' surfaces all have no-slip boundary conditions for the velocity components. These are specified as $u_{f}=0$ on the cylinder wall and $u_{f}=v_{s}$ on the splitter plate wall where $u_{f}$ is the fluid velocity and $v_{s}$ is the plate wall velocity.

2) A uniform flow from the left side to the right side of the domain was considered such that $\operatorname{Re}=\rho U \infty D / \mu=100$, where $\rho$ and $\mu$ are the density and dynamic viscosity of the fluid respectively, $U_{\infty}$ is the free-stream velocity and $D$ is the diameter of the cylinder.

3) At lateral surfaces, the open boundary condition with no viscous stress was imposed which enables the description of boundaries that are open to large volumes of fluid; therefore, the fluid can both enter and leave the domain. This boundary condition prescribes vanishing viscous stresses and can be useful in modeling problems similar to the presented study with high aspect ratio since it does not impose any constraint on the pressure.

4) At downstream outlet, the boundary condition defined through Comsol was called "pressure, no viscous stress" which specifies vanishing viscous stress along with a Dirichlet condition on the pressure. In the presented modeling, the pressure p was set to zero.

The material considered in fluid domain was glycerin with dynamic viscosity of $1.42 \frac{\mathrm{kg}}{\mathrm{m} . \mathrm{s}}$ and density of $1260 \frac{\mathrm{kg}}{\mathrm{m}^{3}}$. The material properties of the solid domain included the Young modulus of $32 \mathrm{MPa}$, Poisson ratio of 0.25 and density of $180 \frac{\mathrm{kg}}{\mathrm{m}^{3}}$. 


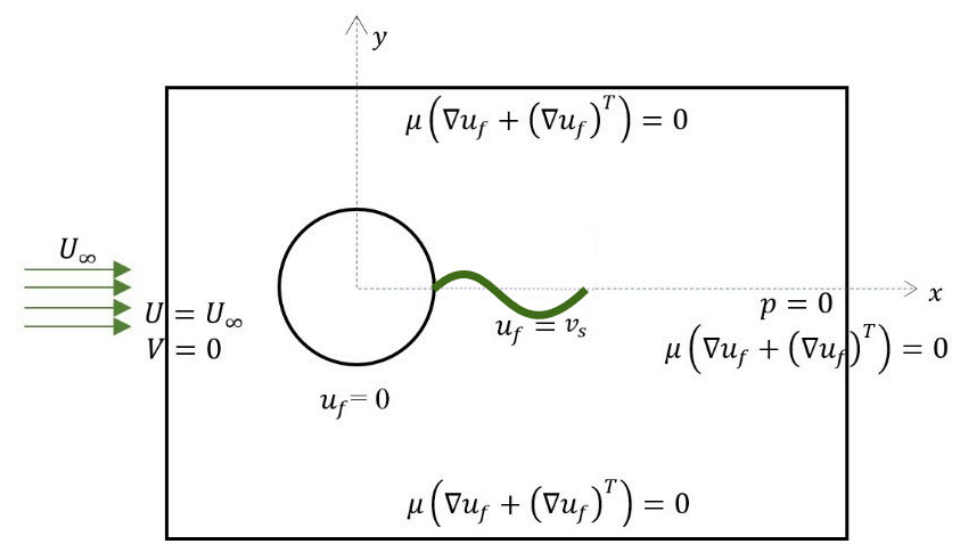

Figure 2: The computational domain and boundary conditions.

The non-dimensional parameters employed in this numerical study including drag coefficient $\left(C_{D}\right)$, the lift coefficient $\left(C_{L}\right)$, the Strouhal number $(S t)$ and the pressure coefficient $\left(C_{P}\right)$ are given by:

$S t=\frac{f_{S} D}{U_{\infty}}$

where $f_{s}$ is the vortex shedding frequency and $U_{\infty}$ denotes the flow velocity.

$$
\begin{aligned}
C_{D} & =\frac{F_{D}}{0.5 \rho U_{\infty}^{2} D} \\
C_{L} & =\frac{F_{L}}{0.5 \rho U_{\infty}^{2} D}
\end{aligned}
$$

where $\rho$ is the fluid density and $F_{D}$ and $F_{L}$ are the fluid forces (i.e., the pressure and viscosity forces) applied on the cylinder, in the streamwise and transverse direction, respectively.

$C_{P}=\frac{P-P_{\infty}}{0.5 \rho U_{\infty}^{2}}$

where $P$ is the static pressure on the cylinder surface and $P_{\infty}$ is the free-stream static pressure.

\section{The numerical methodology and validation}

\subsection{Governing equations and numerical formulation in Comsol Multiphysics}

The commercial software Comsol has a FSI module which utilizes the ALE method to couple the equations of fluid flow formulated based on Eulerian description at a spatial frame, with the equations representing the deformation of the solid material, formulated based on Lagrangian description at a material (reference) frame. The boundaries between the fluid and the solid are subjected to the fluid-structure interaction couplings. Assuming an initial configuration of $\hat{\Omega}$ consisting from the fluid domain $\hat{\Omega}^{f}$, the structural domain $\widehat{\Omega}^{s}$, and an interface where the elastic solid interacts with the fluid represented by $\hat{\Gamma}_{f s i}\left(=\hat{\Omega}^{f} \cap \hat{\Omega}^{s}\right)$. Neglecting the volume forces, the flow field is modeled by means of incompressible and unsteady Navier-Stokes equations in the spatial coordinate system: 


$$
\begin{array}{lc}
\nabla \cdot u_{f}=0 & \text { in } \Omega_{t}^{f} \\
\rho\left(\frac{\partial u_{f}}{\partial t}+\left(u_{f}-u_{m}\right) \cdot \nabla u_{f}\right)=\nabla \cdot\left[-p I+\mu\left(\nabla u_{f}+\left(\nabla u_{f}\right)^{T}\right)\right] & \text { in } \Omega_{t}^{f}
\end{array}
$$

where $\rho$ is the fluid density, $p$ is the pressure, $u_{f}$ is the velocity vector of the fluid in a fixed coordinate system, $u_{m}$ is the velocity vector of the fluid in a moving coordinate system and $I$ is the unit diagonal matrix. The boundary conditions for solving the Navier-Stokes equation are given in section 2. The initial conditions are: $u_{f}=0$ and $p=0$ in $\hat{\Omega}^{f}$ at $t=0$. Because the Navier-Stokes equations are solved in the spatial (deformed) frame while the solid equations are defined in the material (undeformed) frame, a transformation of the force is necessary. This is done according to:

$$
F=f .(d v / d V)
$$

where $d v$ and $d V$ are the mesh element scale factors for the spatial frame and the material (reference) frame, respectively and $f$ and $F$ denote the force in spatial space and the material, respectively.

Formulation of the structural equations is based on a constitutive law given as:

$$
\left(S-S_{0}\right)=C:\left(\varepsilon-\varepsilon_{0}\right)
$$

where $S$ denotes the second Piola-Kirchhoff stress tensor and $C$ denotes is the fourth order elasticity tensor, “:” stands for the double-dot tensor product (or double contraction), $S_{0}$ and $\varepsilon_{0}$ are initial stresses and strains, $\varepsilon$ is the Green-Lagrange strain which is defined by:

$$
\varepsilon=0.5\left[\left(\nabla u_{s}\right)^{T}+\nabla u_{s}+\left(\nabla u_{s}\right)^{T} \nabla u_{s}\right]
$$

where $u_{s}$ is the displacement vector, $(\bullet)^{T}$ represents a transpose. The displacement of surface points on the plate, $u_{s}$ can be calculated from the linear elastic material equation given by:

$\rho^{s} \frac{\partial^{2} u_{s}}{\partial t^{2}}-\nabla . \sigma=F_{V} \quad$ in $\Omega_{t}^{s}$

where $F_{V}$ represents the force per unit volume acting at the fluid-solid interface, $\sigma$ is the Cauchy stress which is relate to second Piola-Kirchhoff stress as: $\sigma=J^{-1} F S F^{T}$ where $F(=I$ $\left.+\nabla u_{s}\right)$ is the deformation gradient tensor and $J=\operatorname{det}(F)$.

The initial conditions for solving equation (10) are: $u_{S}=0$ and $\frac{\partial u_{s}}{\partial t}=0$ in $\widehat{\Omega}^{s}$ at time $t=0$. The fixed boundary condition is assumed for the cylinder surface. Moreover, the plate is fixed at it's intersection with the cylinder.

The spatial frame also deforms with a mesh deformation that is consistent with the displacements $u_{s}$ of the solid material within the solid domains. Comsol computes the new mesh for the fluid domain based on the movement of the structure's boundaries. The Winslow smoothing equations (Knupp, 1999) are used for the moving mesh interface in the simulation which are described as:

$$
\begin{aligned}
& \frac{\partial^{2} \partial X}{\partial x^{2} \partial t}+\frac{\partial^{2} \partial X}{\partial y^{2} \partial t}=0 \\
& \frac{\partial^{2} \partial Y}{\partial x^{2} \partial t}+\frac{\partial^{2} \partial Y}{\partial y^{2} \partial t}=0
\end{aligned}
$$

where $x$ and $y$ are the spatial coordinates of the spatial frame, and $X$ and $Y$ are the reference coordinates of the material frame. 
On the fluid-structure interfaces, $\hat{\Gamma}_{f s i}$, the following geometrical and mechanical conditions must hold:

Firstly, the fluid velocity is calculated from the surface velocity of the structure, i.e.

$v_{f}=v_{s}$

where $v_{s}=\frac{\partial u_{s}}{\partial t}$ is the plate's surface velocity which acts as a moving wall for the fluid domain. Secondly, the load which was exerted by the fluid on the plate surface as a boundary condition at the fluid-structure interface boundary is calculated from:

$\Gamma \cdot n^{f}+\sigma \cdot n^{s}=0$

where $\Gamma\left(=-p I+\mu\left(\nabla u_{f}+\left(\nabla u_{f}\right)^{T}\right)\right)$ is the addition of the pressure with the viscous stress, $\sigma$ is the Cauchy stress tensor in the plate surface and $n^{f}$ and $n^{s}$ are the outward normals corresponding to the fluid and structure, respectively.

After discretization of the above equations, the fluid structure interaction equation can be solved using monolithic method. This method solves the whole FSI problem at every time step through a costly nonlinear system of equations. The steps shown through equations 5-14 are iteratively repeated until a converged solution is obtained at each time step (Comsol, 2012; Ong et al, 2013).

A description of Comsol FEA solvers and finite element scheme used to solve partial differential equations of the problem can be found in our previous work (Abdi et al, 2017). Further information regarding the solution method and efficiency of the solver can also be found from Cheema and Park (2013) and Ong et al (2013) studies.

\subsection{Validation of the finite element model}

Below tables present the results of two comparison studies performed to validate the presented numerical model. Firstly, a plain cylinder and a cylinder with an attached rigid splitter plate modeled with the same computational domain and boundary conditions presented in section 2 were considered. The flow quantities [Strouhal number (St) \& mean drag coefficient $\left(\overline{C_{D}}\right)$ computed from integration of stresses over the cylinder surfaces only] for these two cases were compared with the numerical and experimental results from the literature associated with the two cases. As shown in Table 1, the simulation results are in good agreement with those reported in the literature. 
Table 1: Comparison of characteristic quantities for flow past a circular cylinder with and without rigid splitter plates.

\begin{tabular}{|c|c|c|c|c|c|c|c|}
\hline Test cases & $\begin{array}{c}\text { Flow } \\
\text { quantities }\end{array}$ & $\begin{array}{c}\text { Sudhakar \& } \\
\text { Vengadesan } \\
(2012)\end{array}$ & $\begin{array}{c}\text { Park et } \\
\text { al } \\
(1998)\end{array}$ & $\begin{array}{c}\text { Willia- } \\
\text { mson } \\
(1989)\end{array}$ & $\begin{array}{c}\text { Bao } \\
\text { \& } \\
\text { Tao } \\
(2013)\end{array}$ & $\begin{array}{c}\text { Hwang } \\
\text { et al. } \\
(2003)\end{array}$ & Present \\
\hline Plain circular cylinder & $\begin{array}{c}\text { Mean drag } \\
\text { coefficient } \\
\left(\overline{C_{D}}\right)\end{array}$ & 1.37 & 1.33 & - & 1.335 & 1.34 & 1.33 \\
\cline { 2 - 9 } & $\begin{array}{c}\text { Strouhal } \\
\text { number } \\
(\text { St })\end{array}$ & 0.165 & 0.164 & 0.164 & 0.164 & 0.167 & 0.164 \\
\hline \multirow{2}{*}{$\begin{array}{c}\text { Circular cylinder with one } \\
\text { rigid plate at } \theta_{p}=0^{\circ}\end{array}$} & $\begin{array}{c}\text { Mean drag } \\
\text { coefficient } \\
\left(\overline{C_{D}}\right)\end{array}$ & 1.174 & - & - & - & 1.17 & 1.161 \\
\cline { 2 - 9 } & $\begin{array}{c}\text { Strouhal } \\
\text { number } \\
(S t)\end{array}$ & 0.139 & - & - & - & 0.137 & 0.136 \\
\hline
\end{tabular}

Secondly, the presented numerical methodology was employed to model a cylinder with an attached flexible splitter plate previously studied by Turek \& Hron (2006). The circular cylinder had radius of 0.05 and the length and the thickness of the flexible splitter plate attached to the cylinder at $\theta_{p}=0^{\circ}$ were $L=0.35$ and $h=0.02$, respectively. The cylinder with the attached plate was immersed in a channel of size $L x \times L y=2.5 \times 0.41$ with a parabolic velocity profile at the inlet. A fully developed flow with a mean velocity of $2 \mathrm{~m} / \mathrm{s}$ entered the channel and the Reynolds number was 200. The drag and lift forces applied on the both cylinder and the plate surfaces and the tip displacement of the flexible plate are presented in Table 2. Again, the comparison shows a good agreement between the results from the presented modeling scheme and those from the literature.

Table 2: Comparison of characteristic quantities for flow past a circular cylinder with an attached flexible splitter plate.

\begin{tabular}{|c|c|c|c|}
\hline \multicolumn{2}{|c|}{ Quantities } & Turek \& Horn & Present \\
\hline \multirow{2}{*}{ Drag forces } & Magnitude $(\mathrm{N})$ & $2.22 \pm 149.78$ & $4.5 \pm 150$ \\
\cline { 2 - 4 } & Frequency $(\mathrm{Hz})$ & 10.9 & 10.4 \\
\hline \multirow{2}{*}{ Lift forces } & Magnitude $(\mathrm{N})$ & $457.3 \pm 22.66$ & $454 \pm 25$ \\
\cline { 2 - 4 } & Frequency $(\mathrm{Hz})$ & 5.3 & 5.36 \\
\hline $\begin{array}{c}\text { Plate's tip } \\
\text { displacement (x) }\end{array}$ & Magnitude $(\mathrm{mm})$ & $-2.69 \pm 2.53$ & $-2.7 \pm 2.5$ \\
\cline { 2 - 4 } & Frequency $(\mathrm{Hz})$ & 10.9 & 11 \\
\hline $\begin{array}{c}\text { Plate's tip } \\
\text { displacement (y) }\end{array}$ & Magnitude $(\mathrm{mm})$ & $1.48 \pm 34.38$ & $0.5 \pm 33.5$ \\
\cline { 2 - 4 } & Frequency $(\mathrm{Hz})$ & 5.3 & 5 \\
\hline
\end{tabular}

\subsection{Grid independence test}

This section aims to test the grid independence of the numerical model implemented in the study by investigating the effect of different mesh resolutions on the numerically computed flow quantities of a cylinder with a single flexible splitter plate attached at $\theta_{p}=0^{\circ}$. The finite element model of the fluid domain presented in figure 3 was considered as two subregions: 
1) The inner region: the region near the cylinder and the plate surface in which the velocity gradient is high; hence, a fine mesh with quadrilateral elements was chosen for this region to improve the accuracy of the numerical solution.

2) Outer region in which a coarser triangular mesh was used.

The solid domain was meshed using triangular elements connected to the neighboring elements of the fluid domain.

Table 3 compares the computed flow quantities for four different mesh resolutions. It can be seen that despite the high difference in the number of elements implemented in the four mesh resolutions, the corresponding flow quantities are very close in magnitude. The mesh resolution implemented in case (3) was chosen for the rest of the study since both St and $\overline{C_{D}}$ are converged at this mesh resolution.

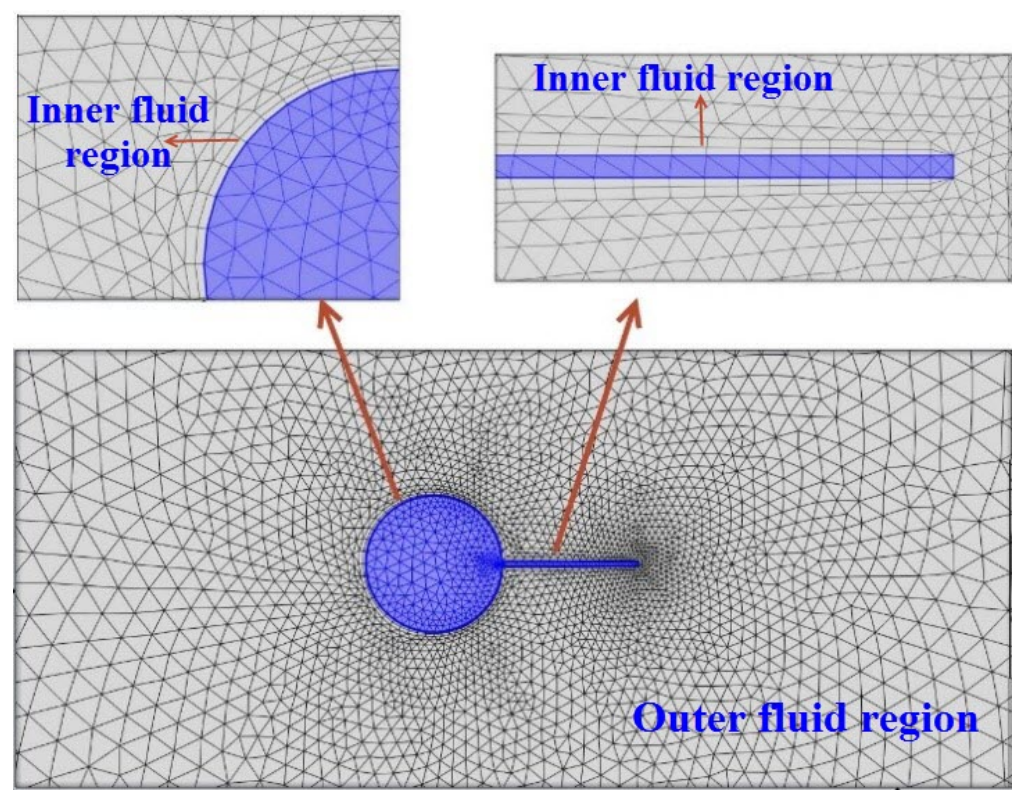

Figure 3: Mesh detail at the interface boundary between the plate wall and fluid domains

Table 3: Grid independence test for the flow past a circular cylinder with one flexible plate attached at $\theta_{p}=0^{\circ} . \overline{C_{D}}$ is computed from integration of stresses over the cylinder surfaces only.

\begin{tabular}{cccc}
\hline Case & Grid elements & $S t$ & $\overline{C_{D}}$ \\
\hline$(1)$ & 2017 & 0.179 & 1.293 \\
$(2)$ & 6446 & 0.177 & 1.292 \\
$(3)$ & 12745 & 0.177 & 1.291 \\
$(4)$ & 30830 & 0.177 & 1.291 \\
\hline
\end{tabular}

\section{Results and discussion}

The three different cases of cylinder with single/multiple attached flexible splitter plates introduced in section 2 of the study (cylinder with single splitter, cylinder with dual-splitters and cylinder with tri-splitters) were simulated using the modeling strategy presented in section 3 and characteristic flow quantities including Strouhal number (St), mean drag coefficient $\left(\overline{C_{D}}\right)$ and fluctuating lift coefficient $\left(C_{L}\right)$ were investigated. The results are 
presented in this section against the location of the plates behind the cylinder identified by attachment angle $\left(\theta_{p}\right)$.

\subsection{Vortex shedding frequency and tip amplitude of the flexible splitter plates}

Figure 4(a) shows the instantaneous streamlines of the flow past a cylinder with a single attached flexible splitter at $\theta_{p}=0^{\circ}$, when the plate moves downward completing a half cycle of oscillation. It can be seen that during this downward motion, the cylinder vortices have grown in a region close to the cylinder. This is in contrast to the corresponding rigid splitter plate case where the growth of the vortices was observed in a region close to the free end of the plate (Abdi et al, 2017). Hence, the vortices of the cylinder with a single flexible splitter resemble the vortices of the plain cylinder (figure $4(\mathrm{~b})$ ). At $\theta_{p}=0^{\circ}, 7.9 \%$ enhancement in St was achieved compared to the plain cylinder. Three mechanisms are responsible for this enhancement; the first mechanism is the application of a propulsive force from the plate to the lower vortex of the cylinder as the flexible plate moves from highest position to the lowest position (figure 4(a)). Hence, the separation of the lower vortex happens faster than the corresponding vortex of the plain cylinder. The second mechanism is related to the other side of the plate where the upper vortex grows. The downward movement of the plate provides the upper vortex with a larger area to grow; hence, it grows faster than the corresponding vortex of the plain cylinder. The third mechanism which has the most influence on flow quantities is the generation of a small vortex near the tip of the flexible splitter plate (called "tip vortex" in this paper). Figure 5(a) shows the instantaneous vorticity contour of the flow over a cylinder with one flexible splitter plate $\left(\theta_{p}=0^{\circ}\right)$, at corresponding times shown in figure 4(a). Two shear layers with opposite senses of rotation are generated downstream of the cylinder. The upper layer has clockwise vorticity and the lower one has counterclockwise vorticity. When the plate moves from the highest position to the middle position, the acceleration is positive and the maximum velocity at the plate is at the middle position. Moving further down, the plate takes a negative acceleration and it reaches a stationary state at the lowest position. When the plate is at its highest position (0T in figure $5 a)$, a shear layer is generated under the plate with a clockwise vorticity as that of the upper vortex of the cylinder. During the downward movement of the plate, the shear layer slips gradually and moves towards the tip of the plate. Consequently, a small vortex is generated on tip of the plate. With further downward movement of the plate, the tip vortex touches the lower cylinder vortex which has an opposite vorticity $(0.4 \mathrm{~T}$ and $0.5 \mathrm{~T}$ in figure $5 \mathrm{a})$. The tip vortex cuts the lower cylinder vortex which has opposite vorticity; hence, the tip vortex contributes in the separation and shedding mechanism of the lower cylinder vortex. Furthermore, in the subsequent upward movement of the plate, the tip vortex touches the upper cylinder vortex. As both these vortices have the same sense of rotation, the tip vortex gets combined with the upper cylinder vortex and enlarges it. A counter clockwise vorticity also gets generated on the other side of the plate when it reaches to the lowest position and similar process occurs in the reverse sense.

It is interesting to know that in the case of the cylinder with a single flexible splitter plate attached at $\theta_{p}=0^{\circ}$, if a rigid plate is used instead of the flexible plate, two tip vortices will be generated as shown figure 5(b); however, these vortices don't touch the shear layers of the cylinder. Hence, they have no significant effect on the separation mechanism of cylinder vortices at the Re number used in this study. 


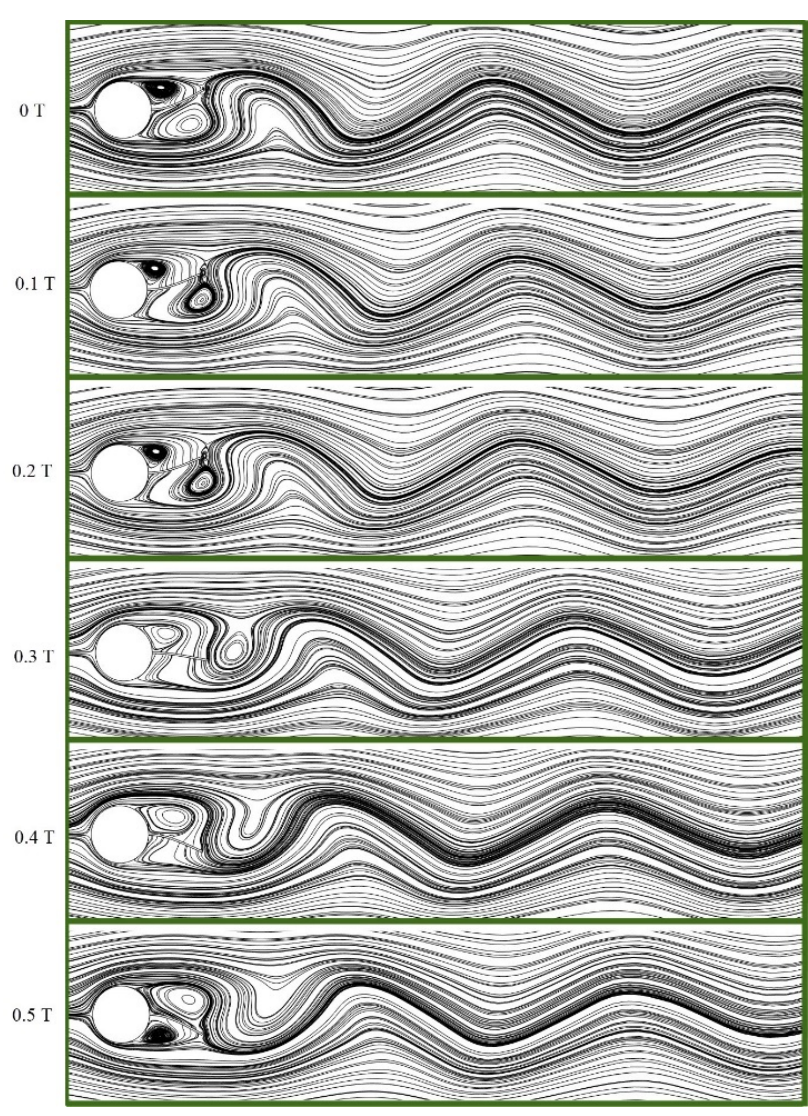

(a)

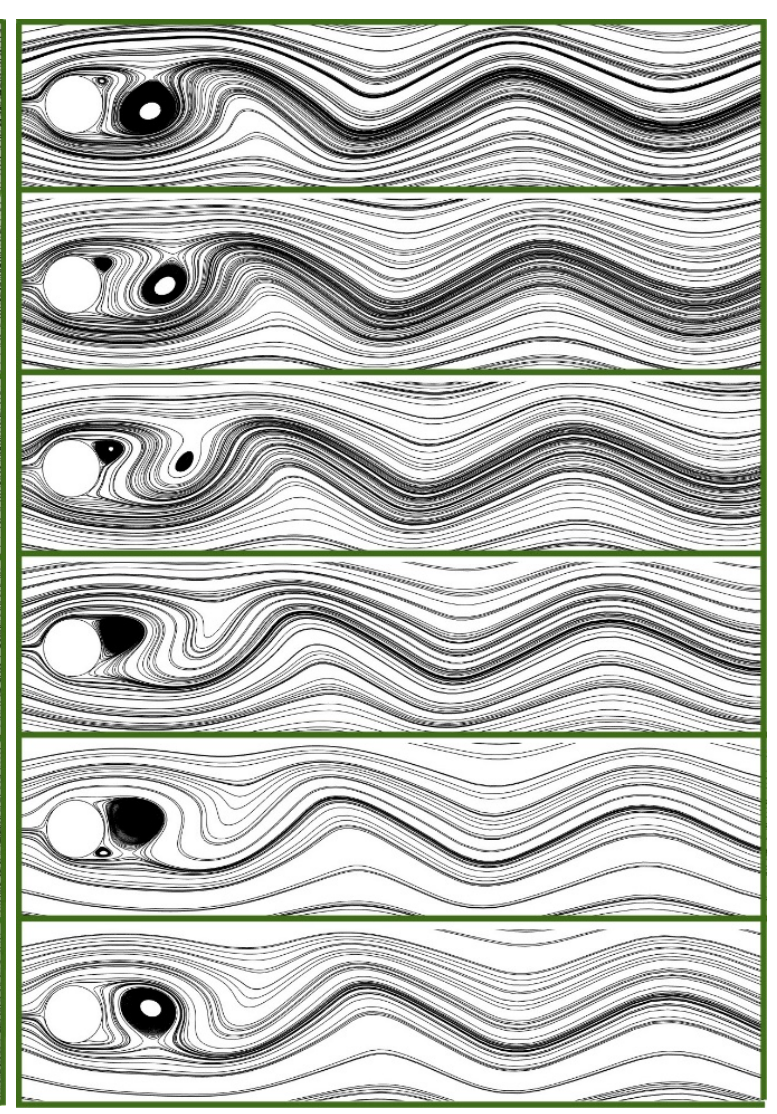

(b)

Figure 4: (a) Instantaneous streamlines for a half cycle of plate oscillation (single flexible plate at $\theta_{p}=0^{\circ}$ ); (b) Instantaneous streamlines for plain cylinder. 


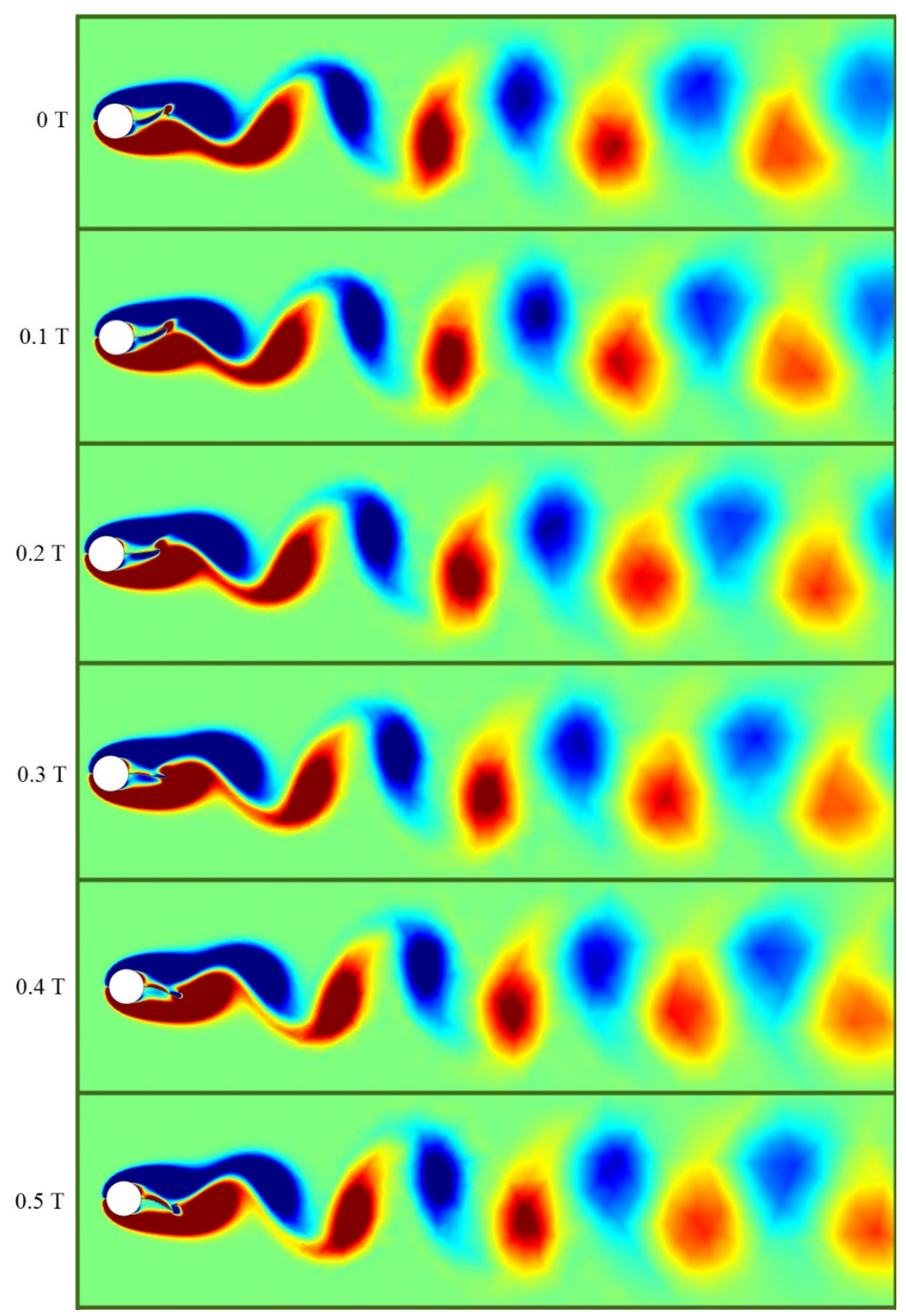

(a)

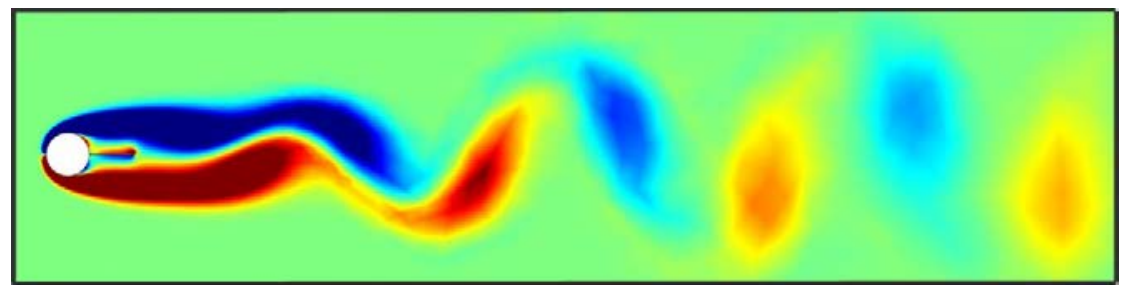

(b)

Figure 5: (a) Instantaneous vorticity contours for a half cycle of plate oscillation (single flexible plate at $\theta_{p}=0^{\circ}$ ); (b) Instantaneous vorticity contour for cylinder with single rigid splitter plate attached at $\theta_{p}=0^{\circ}$ 
Figure 6 shows St against attachment angle $\theta_{p}$ for the cylinder with single/multiple flexible splitter plates. The corresponding plots of plain cylinder and rigid splitter plates (Abdi et al, 2017) are also included for comparison. St was calculated from the period of the lift coefficient. In comparison with rigid splitter plates investigated in a previous study (Abdi et al, 2017), the values of St of the cylinder with flexible splitters was found to be greater than those of the corresponding rigid splitters. It can also be seen from figure 6 that based on the position of the flexible splitter plates (for instance $\theta_{p}>45^{\circ}$ for dual splitters, $\theta_{p}>55^{\circ}$ for tri-splitters and all range of $\theta_{p}$ for single splitter) St can be greater than the corresponding values of the plain cylinder.

In the case of cylinder with single flexible splitter, by increasing $\theta_{p}$, the St number decreased until it reached the corresponding value of the plain cylinder at $\theta_{p}=90^{\circ}$. In this case, the maximum value of St was achieved at $\theta_{p}<15^{\circ}$ which resulted in $7.9 \%$ enhancement compared to the plain cylinder. At small attachment angles of the dual splitters $\left(\theta_{p}<15^{\circ}\right)$, St had small values. However, by further increasing $\theta_{p}$, a sudden rise was observed in the St curve of dual-splitters and St reached the corresponding value of the plain cylinder at $\theta_{p}=$ $30^{\circ}$. Further increasing the $\theta_{p}$ didn't have significant effect on St apart from a slight reduction within $75^{\circ}<\theta_{p}<90^{\circ}$. In the case of flexible tri-splitters, St slightly increased by increasing the attachment angle $\left(0^{\circ}<\theta_{p}<30^{\circ}\right)$ and then a sudden rise was observed by further increasing the attachment angle. In this case, St reached a higher value than the corresponding value of the plain cylinder.

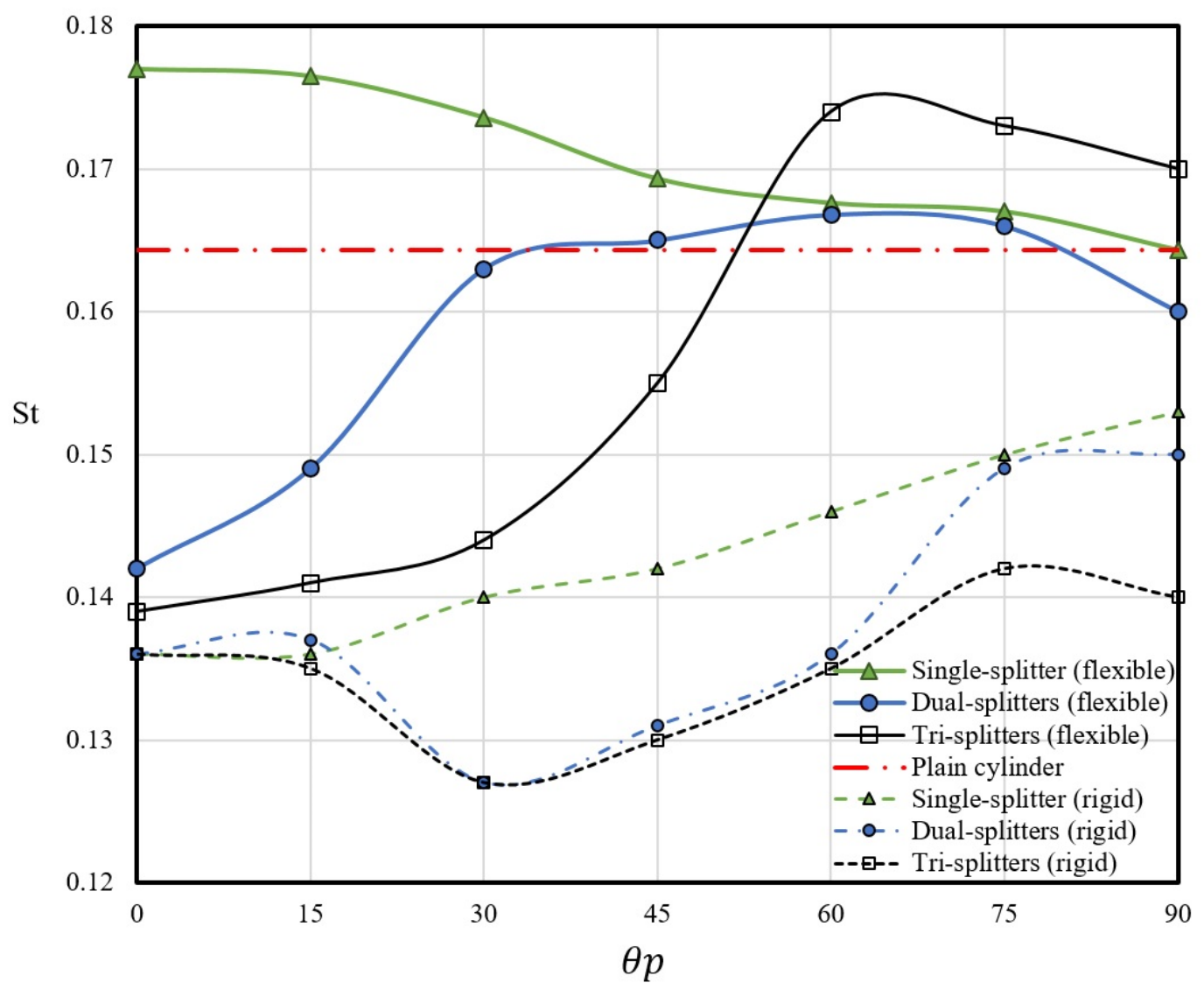

Figure 6: Variation of St number against attachment angle $\left(\theta_{p}\right)$ of splitter plates. The plots corresponding to rigid splitter plates are extracted from (Abdi at al, 2017). 
Figure 7(a) shows the non-dimensional tip amplitude (A/D) of the splitter plates against the attachment angle for all three studied cases of flexible splitters. In the cases of dual-splitters and tri-splitters, the tip amplitudes of the top plates were measured. It can be noticed from the St plot of single flexible splitter that there is a direct relation between the tip amplitude of the plate and the St number. Maximum value of the tip amplitude of the single splitter was observed at $\theta_{p}<15^{\circ}$ which was equal to $0.33 D$. Consequently, a strong vortex was generated near the tip of the plate, resulting the maximum value of $\mathrm{St}$ in this range. By increasing $\theta_{p}$, the tip amplitude gradually decreased, and subsequently, due to the reduction of strength of tip vortex, St decreased. In the case of flexible dual-splitters at $0^{\circ}<\theta_{p}<15^{\circ}$, a curved configuration resembling curved rigid plates with weak oscillations was observed (figure 8). By increasing the $\theta_{p}$ up to $30^{\circ}$, the plates gradually returned back to the former flexible shape with normal oscillations. As a result, a sudden rise was observed within this St range. Similarly, in case of flexible tri-splitters, a curved configuration resembling curved rigid plates was observed at $15^{\circ}<\theta_{p}<30^{\circ}$ and the plates returned to their normal flexible configuration with oscillations when $\theta_{p}$ was increased above this range. Moreover, the middle plate started oscillating with an amplitude higher than those of the lateral plates when $\theta_{p}>45^{\circ}$ (figure $7 \mathrm{~b}$ ) which caused a sudden rise in St number. This effect can explain the direct relationship between the tip amplitude of the middle plate and the St number in the trisplitters observed in figures 6 and 7.

In order to demonstrate the vortex shedding mechanism of flexible splitters at a large attachment angle e.g. $\theta_{p}=60^{\circ}$, instantaneous vorticity contours of all three cases are presented in figure 9 for a half cycle of oscillation showing the movement of the plates from highest position to the lowest position. As can be seen from figures $9 a$ and $9 b$, due to the lower amplitude of the plate at a large attachment angle compared to the single splitter at $\theta_{p}=0^{\circ}$ (figure $4 \mathrm{~b}$ ), weaker vortices were generated at the tip of the plates. Moreover, as the attachment angle increases in single splitter, the influence of tip vortex on the lower cylinder vortex decreased which led to reduction of St, unlike dual-splitters in which both cylinder vortices are affected by the tip vortices.

It can be seen in figure 9c for tri-splitters attached at a large angle $\left(\theta_{p}=60^{\circ}\right)$ that the strong tip vortex of the middle plate interacts with the cylinder vortices. As a result, St has become greater than the St of dual-splitters.

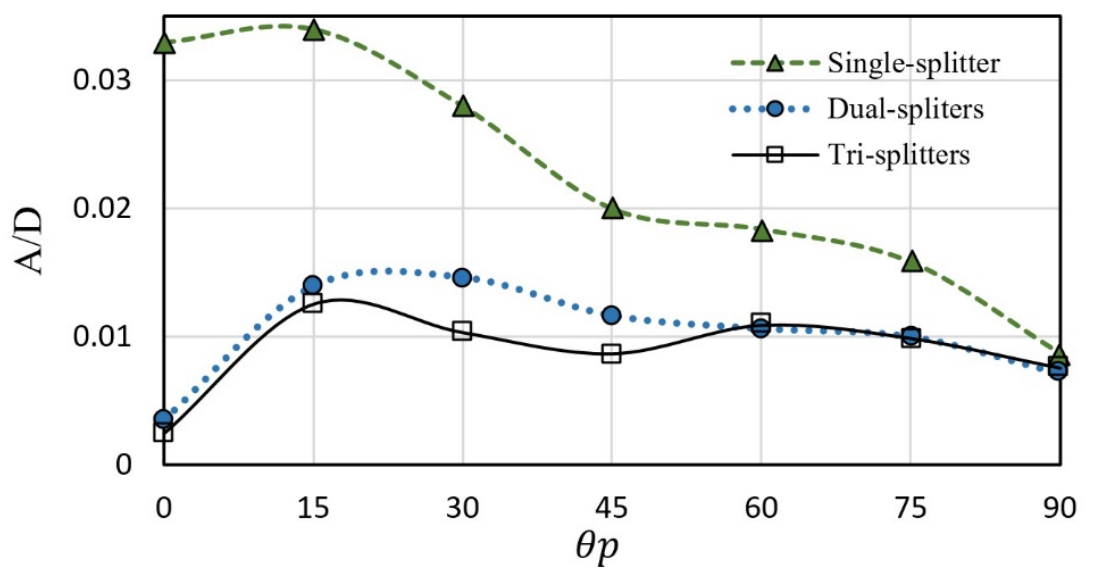

(a) 


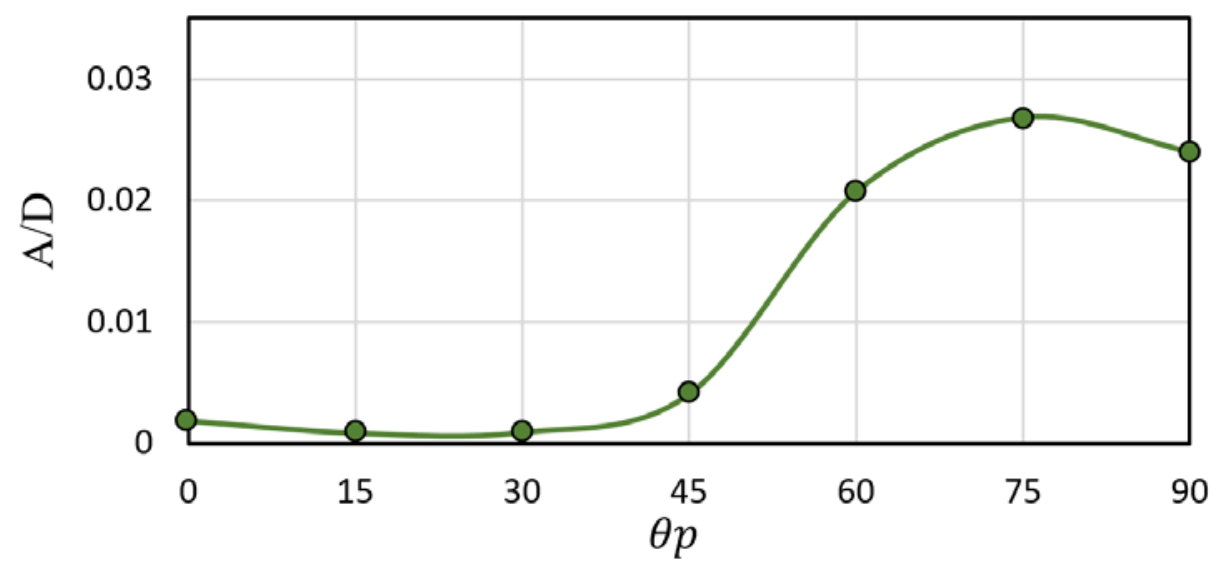

(b)

Figure 7: The non-dimensional plate's tip amplitude, $(A / D)$, against the attachment angle $\left(\theta_{p}\right)$ : (a) top plate (b) middle plate of tri-splitters.

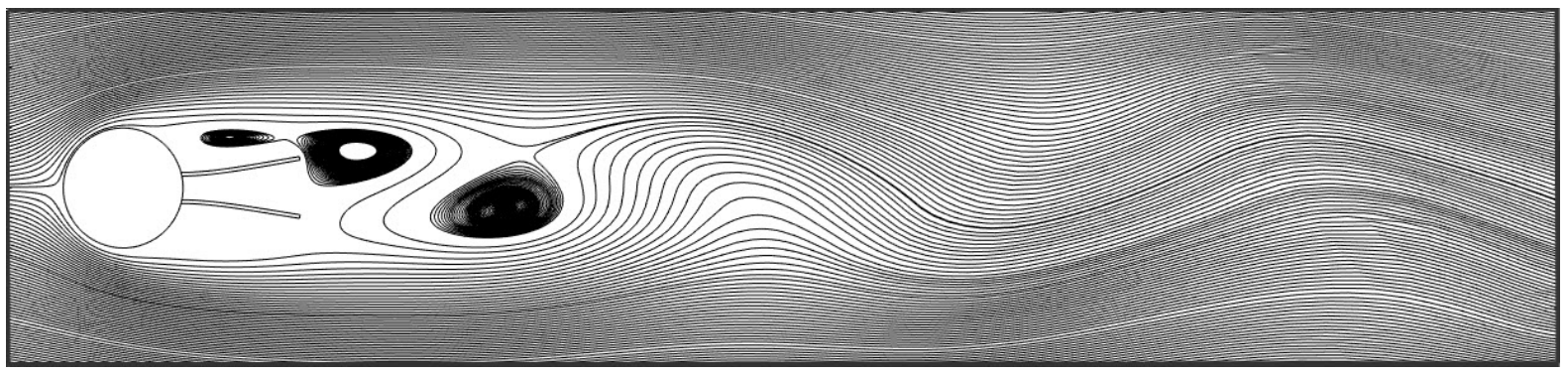

Figure 8: A different mode of oscillation for flexible dual-splitters observed at $0^{\circ}<\theta_{p} \leq 30^{\circ}$. The flexible splitters remained in a constant shape with very weak oscillations resembling curved rigid plates. 


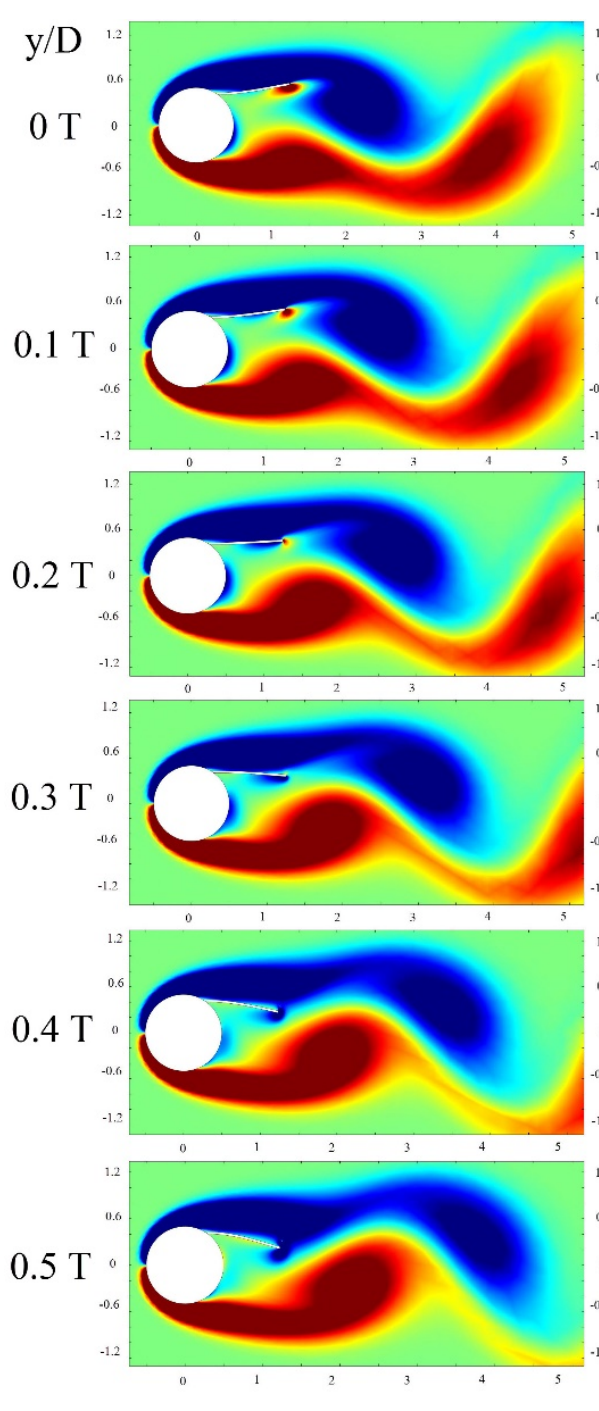

(a)
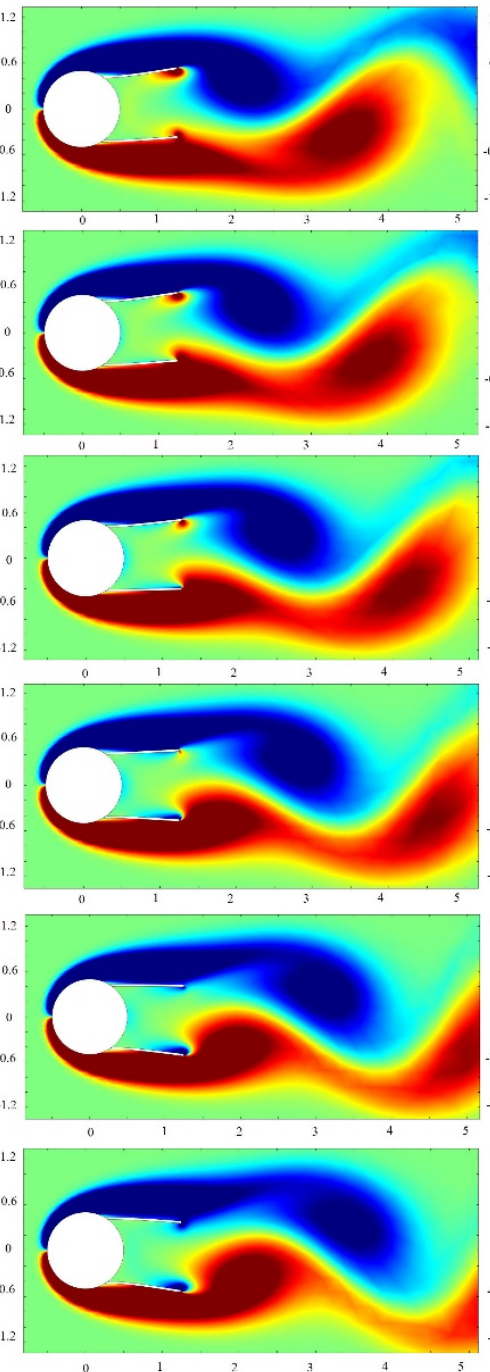

(b)
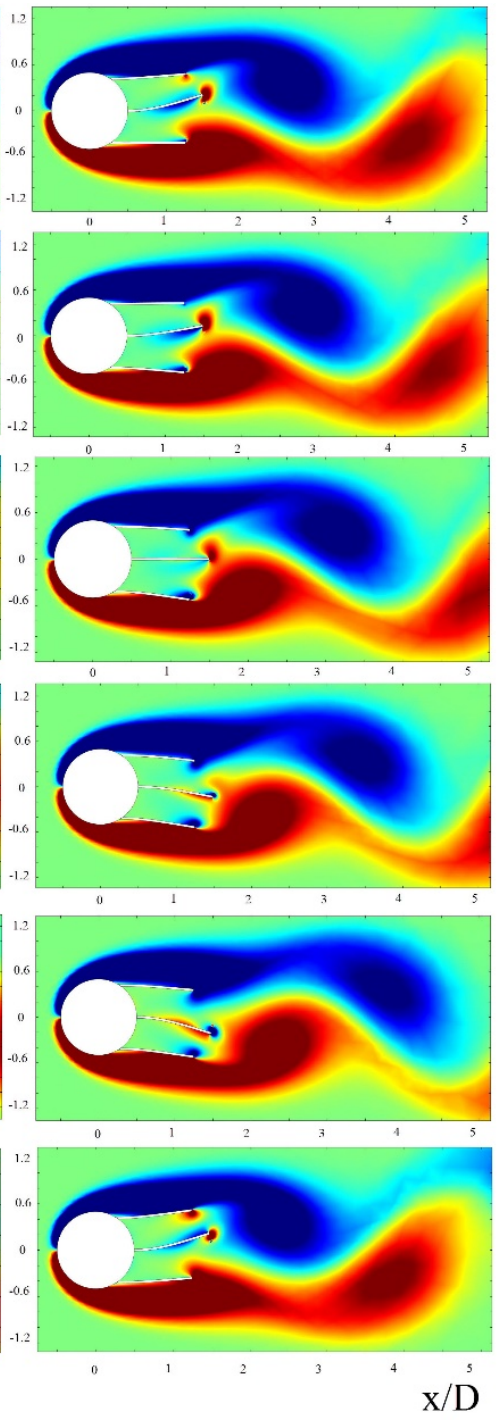

(c)

Figure 9: Instantaneous vorticity contours in a half cycle of plate oscillation at $\theta_{p}=60^{\circ}$ : (a) single splitter, (b) dual-splitters and (c) tri-splitters.

\subsection{Mean drag force and fluctuating lift coefficient}

Figure 10 shows the mean drag coefficient, $\overline{C_{D}}$, against attachment angle of the flexible splitter plate, $\theta_{p}$. The corresponding plots of plain cylinder and rigid splitter plates (Abdi et al, 2017) are also included for comparison. $\overline{C_{D}}$ in figure 10 (a) is computed from integration of stresses over the cylinder surfaces only. $\overline{C_{D t}}$ in figure 10(b) is computed from integration of stresses over both the cylinder and plates' surfaces. It can be seen that the use of flexible splitter plates led to a reduction in $\overline{C_{D}}$ in all three cases compared to plain cylinder. Also in all cases, the minimum $\overline{C_{D}}$ was observed at $\theta_{p}=45^{\circ}$ with $8 \%, 17 \%$ and $20 \%$ reduction in $\overline{C_{D}}$ corresponding to single, dual and tri splitter plates, respectively. Compared to the rigid splitters studied in (Abdi et al, 2017), the presented flexible splitters share a similar profile of $\overline{C_{D}}$, however, an increase in $\overline{C_{D}}$ was observed in the profile of single splitters (when $0^{\circ} \leq$ $\theta_{p} \leq 90^{\circ}$ ), dual-splitters (when $\theta_{p}>15^{\circ}$ ), and tri-splitters (when $\theta_{p}>35^{\circ}$ ). 
It can be seen from figure 10(b) that a similar trend as $\overline{C_{D}}$ was observed for $\overline{C_{D t}}$ in all three cases within $0<\theta_{p}<45^{\circ}$. However, by further increasing $\theta_{p}, \overline{C_{D t}}$ had a higher rate of growth compared to $\overline{C_{D}}$ at $\theta_{p}>45^{\circ}$ in all the simulated cases. This observation can be attributed to the viscosity effect of the plates which has an increasing trend when $\theta_{p}>45$.

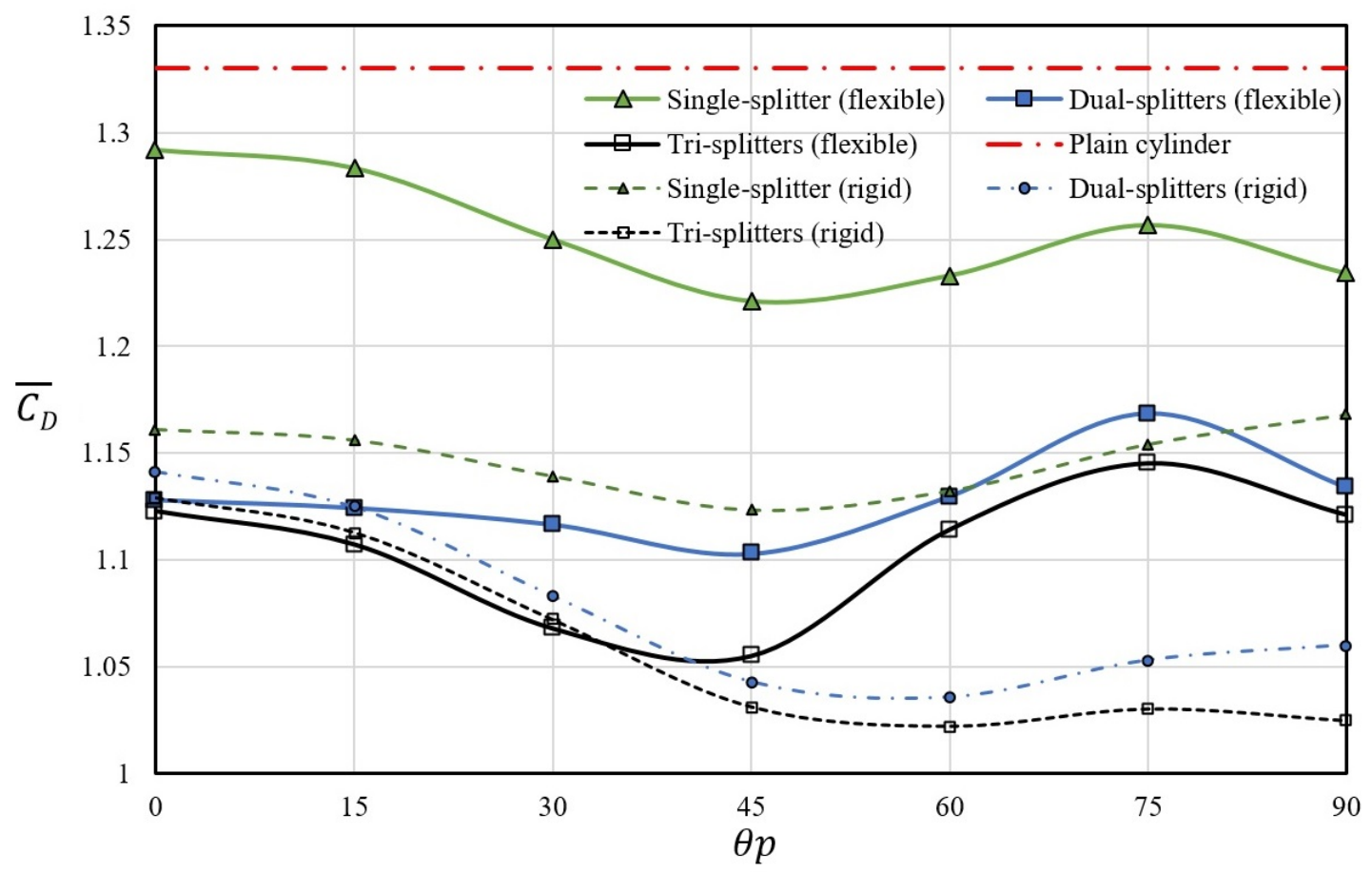

(a)

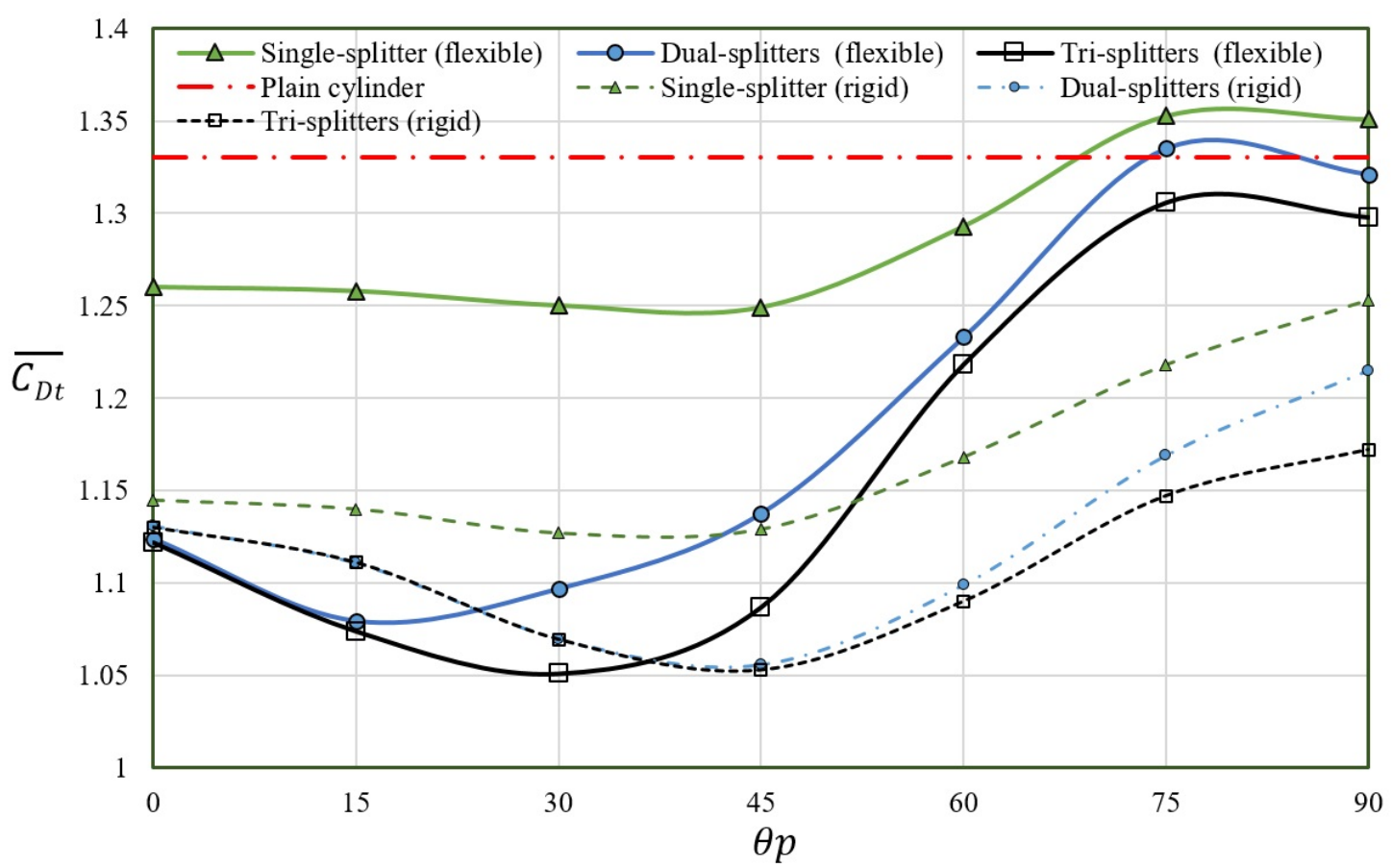

(b)

Figure 10: Mean drag coefficient $C_{D}$, against the attachment angle $\left(\theta_{p}\right)$ of splitter plates: (a) $\overline{C_{D}}$ computed for only the cylinder; (b) $\overline{C_{D t}}$ computed for both the cylinder and plates. The plots corresponding to rigid splitter plates are extracted from (Abdi at al, 2017) 
Figure 11 presents the root-mean-square (r.m.s) value of the fluctuating lift coefficient $\left(C^{\prime}{ }_{L}\right)$ against attachment angle $\theta_{p}$ of flexible splitter plates. The corresponding plots of plain cylinder and rigid splitter plates (Abdi et al, 2017) are also included for comparison. $C^{\prime}{ }_{L}$ represents the influence of the splitter plates on the wake oscillation. As seen for $\overline{C_{D}}$, the graphs of $C^{\prime}{ }_{L}$ shared a similar profile to the corresponding rigid ones studied in (Abdi et al 2017).

The next section of the paper explains the reason for the variation of $\overline{C_{D}}$ and $C^{\prime}{ }_{L}$ based on pressure distribution on the cylinder surface.

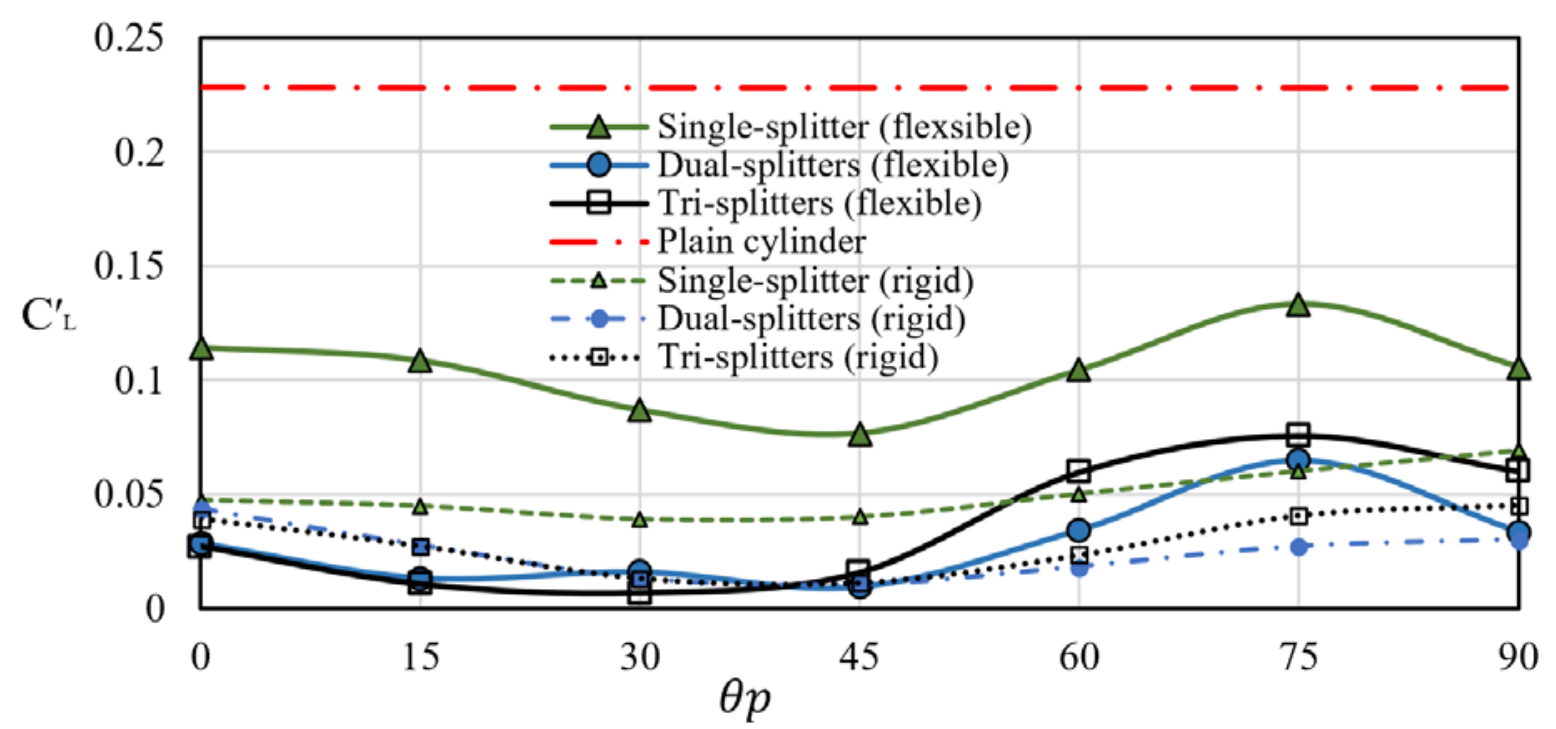

Figure 11: The r.m.s. value of the lift coefficient $\left(C^{\prime}{ }_{L}\right)$, against the attachment angle $\left(\theta_{p}\right)$ of the splitter plates, $\theta_{p} . C_{L}^{\prime}$ is computed from integration of stresses over the cylinder surfaces only. The plots corresponding to rigid splitter plates are extracted from (Abdi at al, 2017)

\subsection{Pressure distribution on the cylinder surface}

The graphs of mean pressure coefficient, $\overline{C_{P}}$, against the angular position of a point of interest around the cylinder, $\theta$, for single and dual flexible splitters are plotted in figure (12) at various $\theta_{p} . \theta$ is calculated from the base point of the cylinder. It should be noted that the variation in $\overline{C_{D}}$ and $C^{\prime}{ }_{L}$ investigated in previous section are directly related to the pressure distribution around the cylinder. Since the curves shared the same profile outside $-120^{\circ} \leq$ $\theta \leq 120^{\circ}$, the graphs in figure (12a) were only plotted in this range, meanwhile, due to symmetric configuration, the graph of figure (12b) were focused in $0^{\circ} \leq \theta \leq 120^{\circ}$. As can be seen from figure (12), the value of $-\overline{C_{P}}$ for all plate positions $\left(\theta_{p}\right)$ were smaller than the associated value of the plain cylinder. Consequently, the value of $\overline{C_{D}}$ (figure 10a) for single splitter and dual splitters in all attachment angles were smaller than the corresponding value of plain cylinder. 


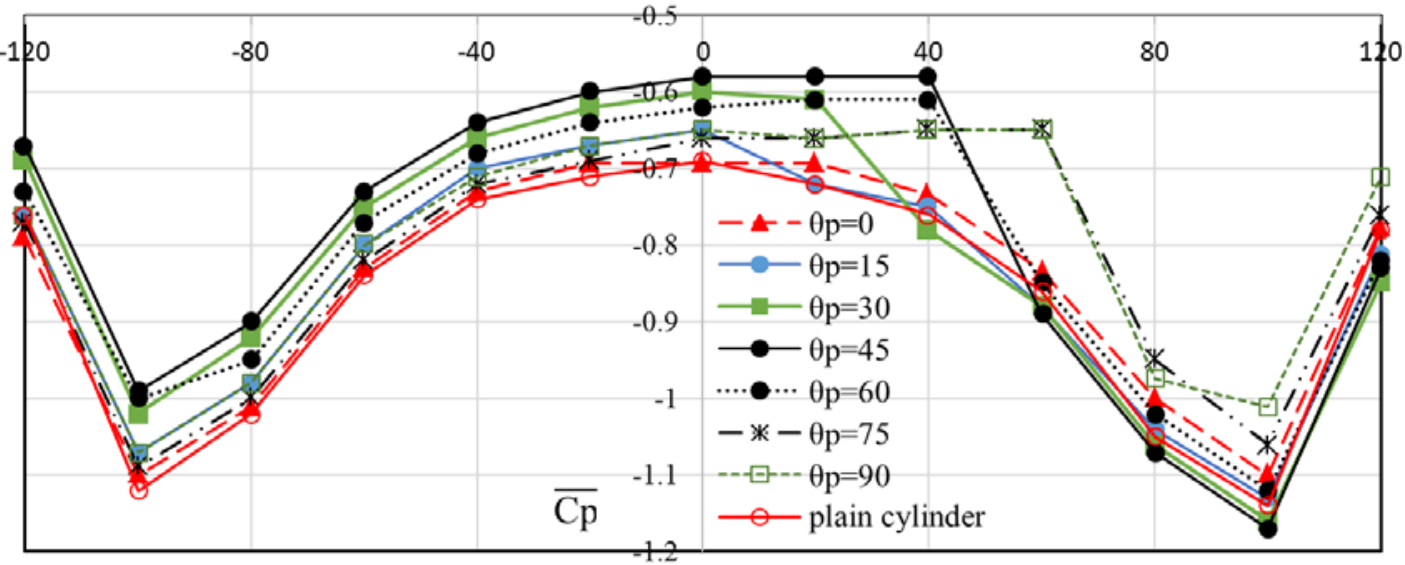

(a)

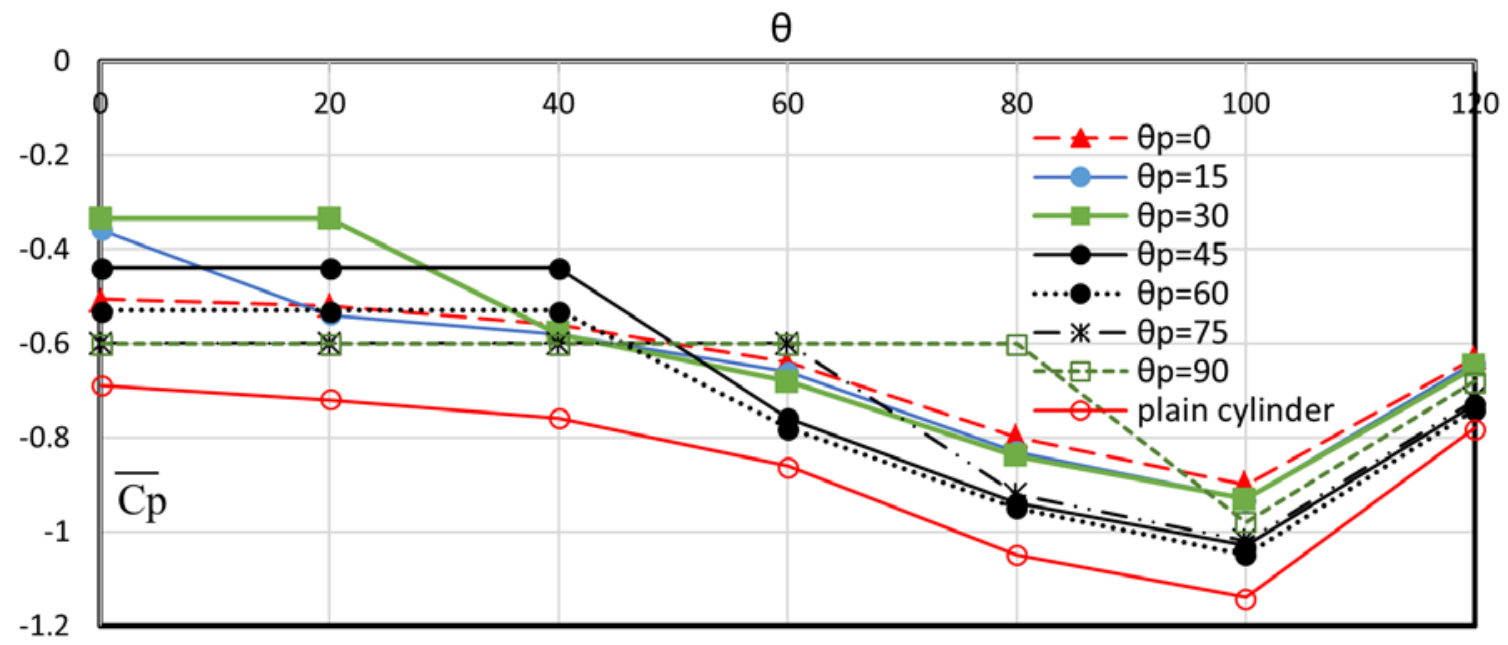

(b)

Figure 12: The mean pressure coefficient distribution, $\overline{C_{P}}$, against the angular location on the cylinder, $\theta$, in various attachment angle $(\theta p)$ of flexible splitter plates at downstream for (a) cylinder with single-splitter (b) cylinder with dual-splitters.

It can be seen in figure 12(b) that in the case of cylinder with dual-splitters, $\overline{C_{P}}$ had a fixed value in the region between the wake centerline and the plate $\left(0<\theta<\theta_{p}\right)$. Similarly, in trisplitters, $\overline{C_{P}}$ is expected to have a fixed value in the region between two adjacent plates $(0<$ $\theta<\theta_{p}$ ). Therefore, the variation of $\overline{C_{D}}$ against attachment angle of tri-splitters case can be justified by the behavior of the mean base pressure coefficient, $\overline{C_{P b}}\left(=\frac{P_{b}-P_{\infty}}{0.5 \rho U_{\infty}^{2}}\right)$ where $P_{b}$ is the static pressure on the cylinder surface at $\theta=0^{\circ}$. The variation of mean base pressure coefficient, $\overline{C_{P b}}$, against the attachment angle for flexible tri-splitters is illustrated in figure 13. Moreover, the plots of $\overline{C_{P b}}$ for the cylinder with dual-splitters as well as the plain cylinder are incorporated into the figure for comparison. In the case of dual/tri-splitter plates at $\theta_{p}=0^{\circ}$, two/three plates on top of each other (in a symmetric position with respect to $x$ axis) were used in the simulation and the mean base pressure coefficient $C_{p b}$ was calculated for a point on cylinder immediately above the attachment point of the upper plate. It can be seen that the plots of dual-splitters and tri-splitters share almost the same profile except within $30^{\circ}<\theta_{p}<60^{\circ}$, therefore, their $\overline{C_{D}}$ is almost equal out of this range. As investigated 
earlier in section 4.1 for flexible tri-splitters, when $30^{\circ}<\theta_{p}<60^{\circ}$, the behavior of the plates is similar to rigid plates with very weak oscillations; hence, in this range $-\overline{C_{P b}}$ is smaller than the corresponding value of dual-splitters.

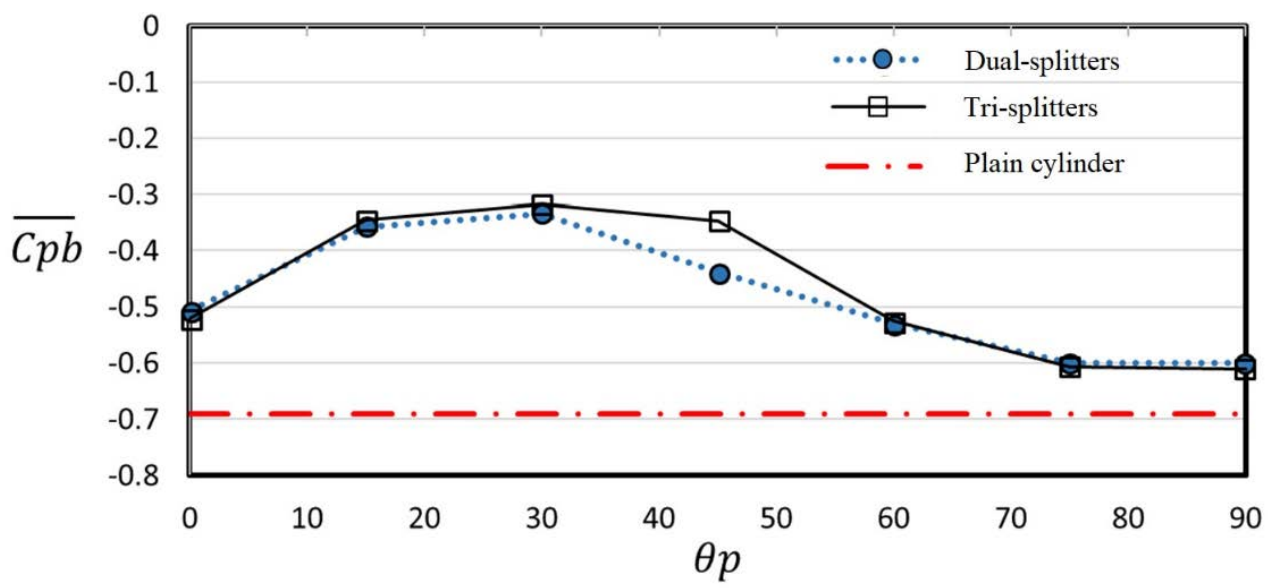

Figure 13: The mean base pressure coefficient, $\overline{C_{P b}}$, against attachment angle $\left(\theta_{p}\right)$, in flexible trisplitters compared with the plain cylinder and cylinder with flexible dual-splitters.

\subsection{Optimum position for locating a piezoelectric polymer}

Piezoelectric polymers can produce electric current when they are placed under mechanical stress. As the electric current produced by the piezoelectric polymer depends on the mechanical strain of the material, the aim is to maximize the applied strain. Figure 14 shows the maximum strain along the plate length against the attachment angle. As can be seen from figure 14 , a maximum strain of 0.018 was observed in single splitter at $\theta_{p}=15^{\circ}$. Also, in trisplitters, a maximum strain of 0.014 was observed in the middle plate at $\theta_{p}=75^{\circ}$. However, considering the fact that in the tri-splitters, less drag coefficient was observed (figure 10), we recommend this as the best position for locating a piezoelectric polymer in terms of harvesting power along with reducing drag forces. 


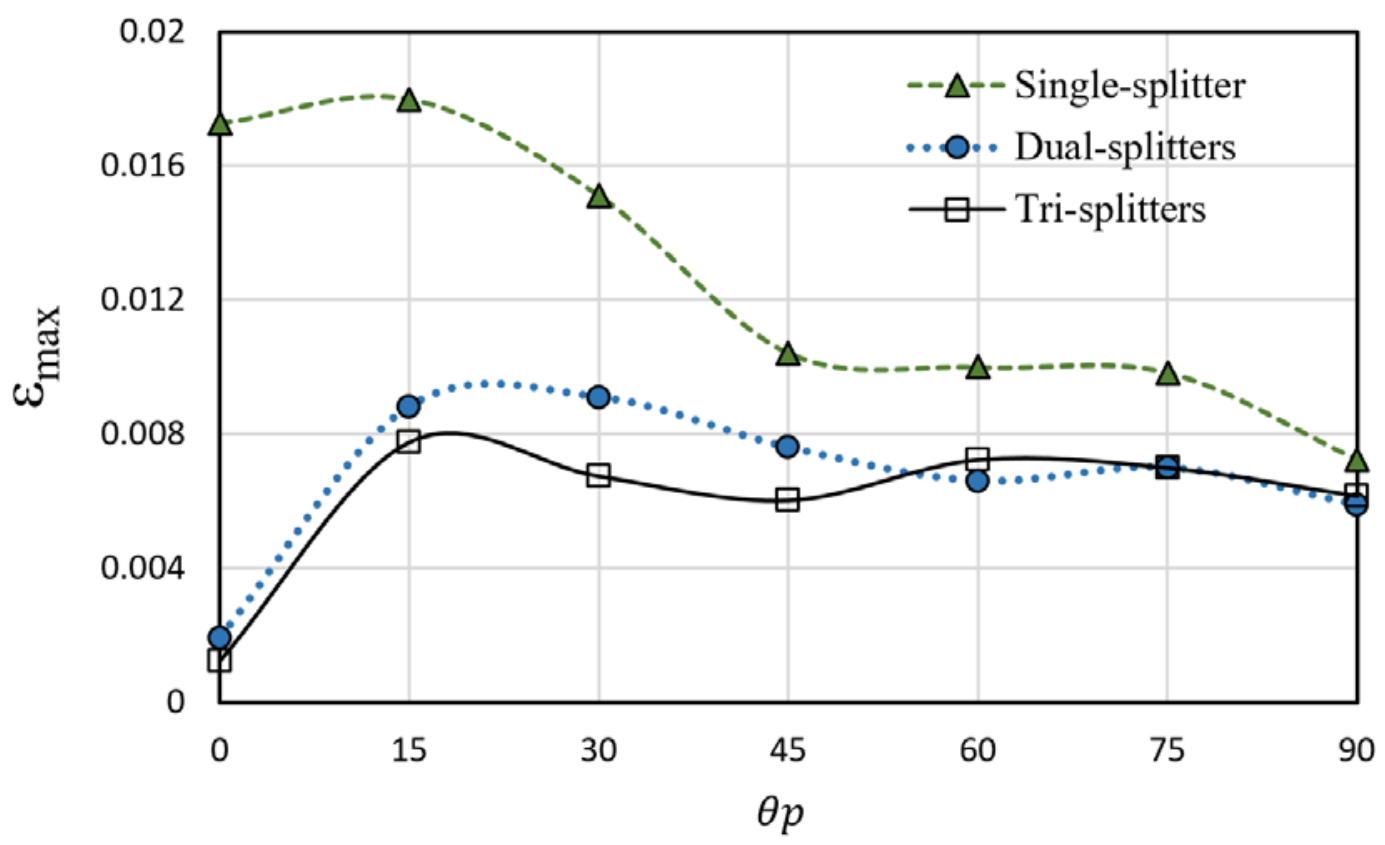

(a)

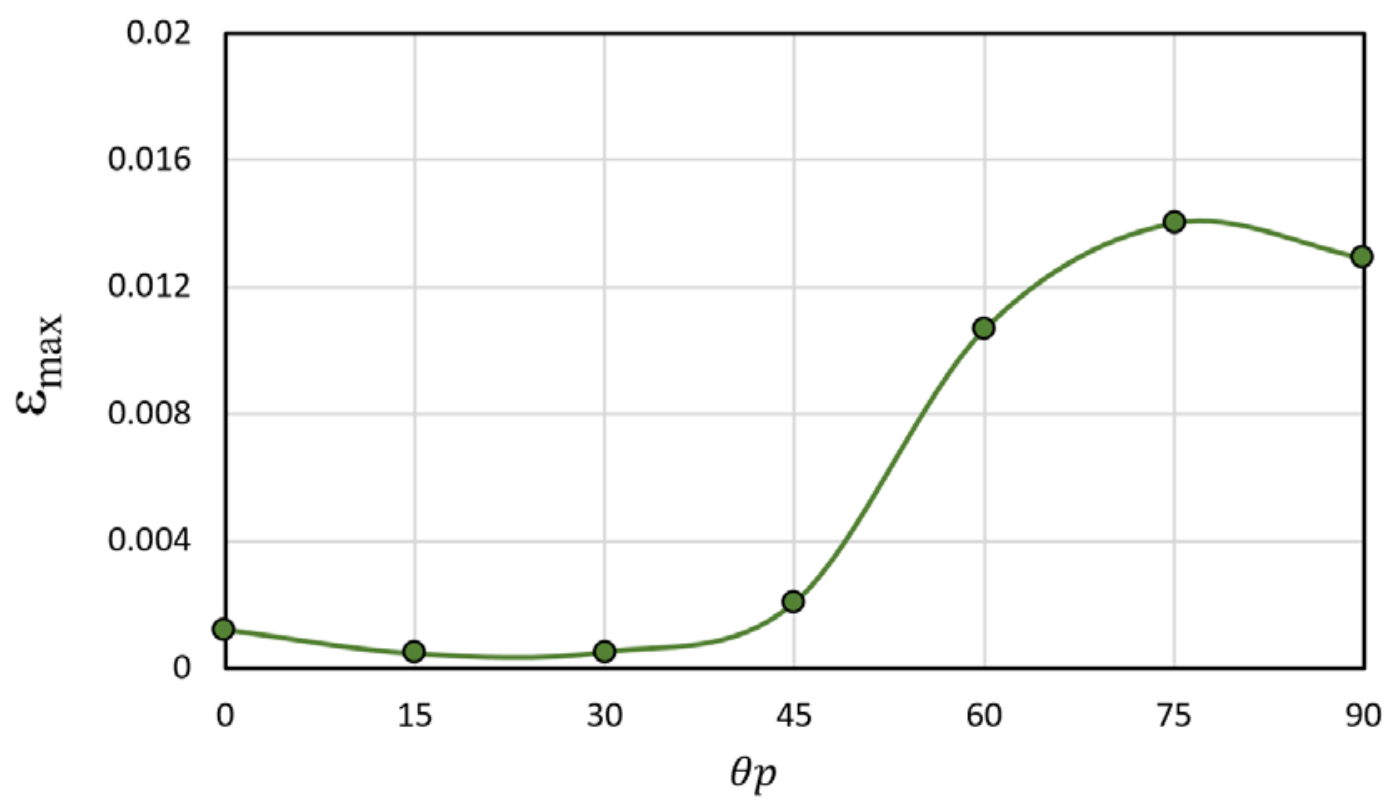

(b)

Figure 14: The maximum strain $\left(\varepsilon_{\max }\right)$ along the plate length, against the attachment angle $\left(\theta_{p}\right)$ : (a) single splitter and the top plate in dual and tri-splitters; (b) middle plate in tri-splitters.

\section{Summary and conclusions}

Wake control of a circular cylinder using attached flexible splitter plates was studied in this paper. A numerical study of fluid-structure interaction for the cylinder and the splitter plates subjected to 2D laminar flow was presented in the paper. The splitter plates had a fixed length equal to the cylinder diameter and the location of horizontal plates (attachment angle) was varied. Three different cases implementing flexible splitter plates for wake control were studied and compared, including single splitter, dual-splitters and tri-splitters. The numerical 
results were also compared to corresponding results of rigid splitter plates from a previous study in which the fluid structure interaction was neglected. The use of piezoelectric polymers can offer energy harvesting from the oscillations of the flexible plates. The maximum strain along the length of the splitter plates was measured and the best position for installing a piezoelectric polymer in terms of energy harvesting was investigated.

In all three cases studied in this paper, the use of flexible splitter plates attached to the cylinder resulted in reducing the drag coefficient, which is a similar to that seen for rigid splitter plates in our previous study. However, compared to the corresponding rigid splitter plates, a rise in drag coefficient of the cylinder with flexible splitter plates was observed. A similar effect was observed when comparing the fluctuating lift coefficient of the flexible splitter plates studied in this paper with rigid splitter plates investigated in our previous study. Also, in contrast to the use of rigid splitter plates which led to a reduction in St, the use of flexible plates resulted in increasing the St number compared to the plain cylinder.

Increasing the number and the attachment angle of flexible splitter plates had crucial effects on drag coefficient, Strouhal number and r.m.s. value of the lift coefficient. In all three cases, the minimum value of $\overline{C_{D}}$ was achieved at the attachment angle of $\theta_{p}=45^{\circ}$ which resulted in $8 \%, 17 \%$ and $20 \% C_{D}$ reduction compared to plain cylinder corresponding to single splitter, dual splitters and tri-splitters, respectively. Within the three cases studied in this paper while considering both energy harvesting and drag reduction, the middle plate of the tri-splitters attached to the cylinder at $\theta_{p}=75^{\circ}$ was found as the best position for locating a piezoelectric polymer.

To sum up, the results of this study suggest that the drag on cylinder, vortex shedding frequency and fluctuations of lift coefficient can be effectively reduced by attaching one or more flexible splitter plates placed in appropriate position. Further work is required to study the effect of other parameters such as the Reynolds number, mass ratio and elastic properties of the splitter plates.

\section{References}

Abdi, R., Rezazadeh, N., \& Abdi, M. (2017). Reduction of fluid forces and vortex shedding frequency of a circular cylinder using rigid splitter plates. European Journal of Computational Mechanics, 26(3), 225-244.

Akaydın, H. D., Elvin, N., \& Andreopoulos, Y. (2010). Wake of a cylinder: a paradigm for energy harvesting with piezoelectric materials. Experiments in Fluids, 49(1), 291-304.

Akilli, H., Sahin, B., \& Tumen, N. F. (2005). Suppression of vortex shedding of circular cylinder in shallow water by a splitter plate. Flow Measurement and Instrumentation, 16(4), 211-219.

Allen, J. J., \& Smits, A. J. (2001). Energy harvesting eel. Journal of fluids and structures, 15(3), 629-640.

Apelt, C. J., West, G. S., \& Szewczyk, A. A. (1973). The effects of wake splitter plates on the flow past a circular cylinder in the range $104<\mathrm{R}<5 \times 104$. Journal of Fluid Mechanics, 61(01), 187-198.

Apelt, C. J., \& West, G. S. (1975). The effects of wake splitter plates on bluff-body flow in the range $104<\mathrm{R}<$ 5× 10 4. Part 2. Journal of Fluid Mechanics, 71(01), 145-160.

Argentina, M., \& Mahadevan, L. (2005). Fluid-flow-induced flutter of a flag. Proceedings of the National academy of Sciences of the United States of America, 102(6), 1829-1834.

Assi, G. R., Bearman, P. W., \& Kitney, N. (2009). Low drag solutions for suppressing vortex-induced vibration of circular cylinders. Journal of Fluids and Structures, 25(4), 666-675. 
Bao, Y., \& Tao, J. (2013). The passive control of wake flow behind a circular cylinder by parallel dual plates. Journal of Fluids and Structures, 37, 201-219.

Bearman, P. W. (1965). Investigation of the flow behind a two-dimensional model with a blunt trailing edge and fitted with splitter plates. Journal of Fluid Mechanics, 21(02), 241-255.

Brown, P. N., Hindmarsh, A. C., \& Petzold, L. R. (1994). Using Krylov methods in the solution of large-scale differential-algebraic systems. SIAM Journal on Scientific Computing, 15(6), 1467-1488.

Cardell, G. S. (1993). Flow past a circular cylinder with a permeable wake splitter plate (Doctoral dissertation, California Institute of Technology).

Cheema, T. A., \& Park, C. W. (2013). Numerical investigation of hyperelastic wall deformation characteristics in a micro-scale stenotic blood vessel. Korea-Australia Rheology Journal, 25(3), 121-127.

Comsol Multiphysics v. 4.3a, User’s Manual 2012.

Connell, B. S., \& Yue, D. K. (2007). Flapping dynamics of a flag in a uniform stream. Journal of fluid mechanics, 581, 33-67.

Eloy, C., Lagrange, R., Souilliez, C., \& Schouveiler, L. (2008). Aeroelastic instability of cantilevered flexible plates in uniform flow. Journal of Fluid Mechanics, 611, 97-106.

Gerrard, J. H. (1966). The mechanics of the formation region of vortices behind bluff bodies. Journal of Fluid Mechanics, 25(02), 401-413.

Gu, F., Wang, J. S., Qiao, X. Q., \& Huang, Z. (2012). Pressure distribution, fluctuating forces and vortex shedding behavior of circular cylinder with rotatable splitter plates. Journal of fluids and structures, 28, 263278.

Hindmarsh, A. C., Brown, P. N., Grant, K. E., Lee, S. L., Serban, R., Shumaker, D. E., \& Woodward, C. S. (2005). SUNDIALS: Suite of nonlinear and differential/algebraic equation solvers. ACM Transactions on Mathematical Software (TOMS), 31(3), 363-396.

Hwang, J. Y., Yang, K. S., \& Sun, S. H. (2003). Reduction of flow-induced forces on a circular cylinder using a detached splitter plate. Physics of Fluids (1994-present), 15(8), 2433-2436.

Knupp, P. M. (1999). Winslow smoothing on two-dimensional unstructured meshes. Engineering with Computers, 15(3), 263-268.

Kwon, K., \& Choi, H. (1996). Control of laminar vortex shedding behind a circular cylinder using splitter plates. Physics of Fluids (1994-present), 8(2), 479-486.

Lee, J., \& Lee, S. (2013). Fluid-structure interaction for the propulsive velocity of a flapping flexible plate at low Reynolds number. Computers \& Fluids, 71, 348-374.

Mansy, H., Yang, P. M., \& Williams, D. R. (1994). Quantitative measurements of three-dimensional structures in the wake of a circular cylinder. Journal of Fluid Mechanics, 270, 277-296.

Nakamura, Y. (1996). Vortex shedding from bluff bodies with splitter plates. Journal of Fluids and Structures, 10(2), 147-158.

Ong, C., Dokos, S., Chan, B., Lim, E., Al Abed, A., Osman, N. \& Lovell, N. H. (2013). Numerical investigation of the effect of cannula placement on thrombosis. Theoretical Biology and Medical Modelling, 10(1), 1.

Ozono, S. (2003). Vortex suppression of the cylinder wake by deflectors. Journal of wind engineering and industrial aerodynamics, 91(1), 91-99.

Posdziech, O., \& Grundmann, R. (2007). A systematic approach to the numerical calculation of fundamental quantities of the two-dimensional flow over a circular cylinder. Journal of Fluids and Structures, 23(3), 479-499.

Roshko, A. (1954). On the drag and shedding frequency of two-dimensional bluff bodies (No. TN3169). NATIONAL AERONAUTICS AND SPACE ADMINISTRATION WASHINGTON DC.

Roshko, A. (1955). On the wake and drag of bluff bodies. Journal of the Aeronautical Sciences (Institute of the Aeronautical Sciences), 22(2).

Sawada, T., \& Hisada, T. (2007). Fluid-structure interaction analysis of the two-dimensional flag-in-wind problem by an interface-tracking ALE finite element method. Computers \& fluids, 36(1), 136-146. 
Schenk, O., \& Gärtner, K. (2004). Solving unsymmetric sparse systems of linear equations with PARDISO. Future Generation Computer Systems, 20(3), 475-487.

Schenk, O., \& Gärtner, K. (2006). On fast factorization pivoting methods for sparse symmetric indefinite systems. Electronic Transactions on Numerical Analysis, 23(1), 158-179.

Souli, M., Ouahsine, A., \& Lewin, L. (2000). ALE formulation for fluid-structure interaction problems. Computer methods in applied mechanics and engineering, 190(5), 659-675.

Sudhakar, Y., \& Vengadesan, S. (2012). Vortex shedding characteristics of a circular cylinder with an oscillating wake splitter plate. Computers \& Fluids, 53, 40-52.

Sumer, B. M., \& Fredsøe, J. (1997). Hydrodynamis around cylindrical structures (No. 12). World Scientific.

Taylor, G. W., Burns, J. R., Kammann, S. M., Powers, W. B., \& Wel, T. R. (2001). The energy harvesting eel: a small subsurface ocean/river power generator. Oceanic Engineering, IEEE Journal of, 26(4), 539-547.

Tian, F. B., Luo, H., Zhu, L., Liao, J. C., \& Lu, X. Y. (2011). An efficient immersed boundary-lattice Boltzmann method for the hydrodynamic interaction of elastic filaments. Journal of computational physics, 230(19), 7266-7283.

Turek, S., \& Hron, J. (2006). Proposal for numerical benchmarking of fluid-structure interaction between an elastic object and laminar incompressible flow. In Fluid-structure interaction (pp. 371-385). Springer, Berlin, Heidelberg.

Unal, M. F., \& Rockwell, D. (1988). On vortex formation from a cylinder. Part 1. The initial instability. Journal of Fluid Mechanics, 190, 491-512.

Williamson, C. H. K. (1989). Oblique and parallel modes of vortex shedding in the wake of a circular cylinder at low Reynolds numbers. Journal of Fluid Mechanics, 206, 579-627.

Wu, J., Shu, C., \& Zhao, N. (2014). Numerical study of flow control via the interaction between a circular cylinder and a flexible plate. Journal of Fluids and Structures, 49, 594-613.

Yuan, H. Z., Niu, X. D., Shu, S., Li, M., \& Yamaguchi, H. (2014). A momentum exchange-based immersed boundary-lattice Boltzmann method for simulating a flexible filament in an incompressible flow. Computers \& Mathematics with Applications, 67(5), 1039-1056. 